\title{
O)
}

AIAA 2001-0249

\section{Advanced Aerodynamic Design of Passive Porosity Control Effectors}

C.A. Hunter, S.A. Viken, R.M. Wood, and S.X.S. Bauer NASA Langley Research Center

Hampton, Virginia

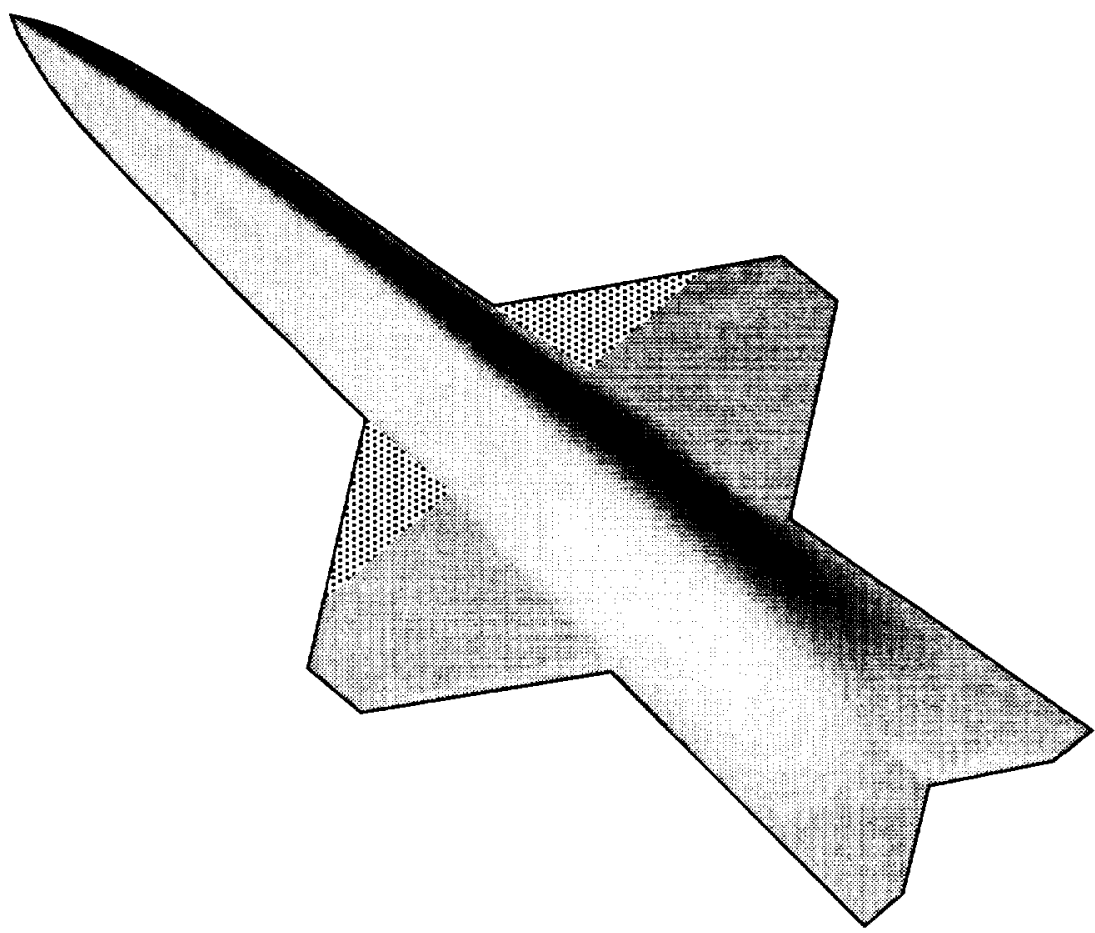

\section{9th AIAA Aerospace Sciences Meeting \& Exhibit 8-11 January 2001 / Reno, NV}





\title{
ADVANCED AERODYNAMIC DESIGN OF PASSIVE POROSITY CONTROL EFFECTORS
}

\author{
Craig A. Hunter", Sally A. Viken ${ }^{* *}$, Richard M. Wood, and Steven X.S. Bauer ${ }^{* \dagger}$ \\ NASA Langley Research Center \\ Hampton, Virginia
}

\begin{abstract}
This paper describes aerodynamic design work aimed at developing a passive porosity control effector system for a generic tailless fighter aircraft. As part of this work, a computational design tool was developed and used to layout passive porosity effector systems for longitudinal and lateral-directional control at a lowspeed, high angle of attack condition. Aerodynamic analysis was conducted using the NASA Langley computational fluid dynamics code USM 3 D, in conjunction with a newly formulated surface boundary condition for passive porosity. Results indicate that passive porosity effectors can provide maneuver control increments that equal and exceed those of conventional aerodynamic effectors for low-speed, high-alpha flight, with control levels that are a linear function of porous area. This work demonstrates the tremendous potential of passive porosity to yield simple control effector systems that have no external moving parts and will preserve an aircraft's fixed outer moldline.
\end{abstract}

\section{Introduction}

Since 1990, the aeronautics research community has explored novel concepts for aircraft shaping and aerodynamic control $|1-9|$. These concepts seek to increase aerodynamic performance, improve survivability, and reduce weight and complexity by eliminating the breaks, gaps, hinges, and mechanical components found in conventional aircraft control effector designs and replacing them with a combination of blended control surfaces, smart materials, and flow control actuators. The result of this design approach is a continuous or even fixed outer moldline aircraft that is able to morph its effective aerodynamic shape and provide maneuver control in a smooth, continuous, organic manner, similar to the methods birds and fish use in nature. While promising, much of the recent research in this area has focused on fundamental flow physics and material/actuator development, and many control effector concepts remain unproven at realistic full scale flight conditions at this time [10-13].

One exception in this area is the passive porosity concept, which was originally developed in the early 1980 s as a means of shock - boundary layer interaction control $|14-19|$. Based on the initial control effector research reported by Bauer $|17|$. passive porosity has evolved into an extensively tested and well proven aerodynamic flow control technology with a wide range of capabilities and applications $[1.20-23]$. The passive porosity concept consists of a porous outer surface, a plenum, and a solid inner surface. as shown in Figure 1. Pressure differences between high- and low-pressure regions on the outer surface "communicate" through the plenum, thereby modifying the pressure loading on the outer surface. In concert with this pressure communication. there is a small amount of mass transfer into and out of the plenum that changes the effective aerodynamic shape of the outer surface.

When regions with a large pressure difference are connected by a passive porosity system, it has the potential to act as a control effector. For example, connecting a low-pressure region on the upper surface of an aircraft with a corresponding high-pressure region on the lower surface of the aircraft would reduce the pressure difference between the two regions, decreasing the local normal force and translating the center of pressure. Applied strategically to different areas of an aircraft, as shown in Figure 2, this can result in an extremely powerful control effector system capable of generating a variety of forces and moments. The general idea would be to equip an aircraft with a number of porous cavities and interconnected plenums that could be controlled and actuated by valves or other simple devices, as illustrated in Figure 3. Compared to a traditional control effector such as a trailing edge flap, passive porosity has no external moving parts. it preserves the vehicle outer moldline, and it should provide better performance by generating a control force that varies linearly with vehicle lift in a predictable manner [1].

In the past, passive porosity has been applied to existing aircraft configurations, working within the limitations and constraints of aircraft designed to use conventional control effectors. While much of this work has been successful, the full potential of passive porosity control effectors can best be explored with a clean-sheet approach - one using an aircraft design that is suitable for advanced aerodynamic control effectors and can be configured to exploit the working principles of passive

Copyright $C 2001$ by the American Institute of Aeronautics and Astronautics. Inc. No copyright is asserted in the Inited States under Title 17. US Code. The US Government has a rovalty-free license to exercise all rights under the copyright claimed herein for government purposes. All other rights are reserved by the copyright owner. 
porosity. This paper describes aerodynamic design work aimed at developing a passive porosity control effector system for such an aircraft, based on a simple tailless fighter concept. In the current investigation, the focus has been narrowed to address longitudinal and lateral-directional maneuver control at low-speed, high angle of attack conditions. Future studies will extend this design work to more general maneuvering in other regimes.

\section{Nomenclature}

$\begin{array}{ll}\alpha, \text { AOA } & \text { Angle of Attack. degrees } \\ \text { BL, } y & \text { Butt Line (spanwise) Coordinate, inches } \\ c & \text { Aerodynamic Chord (local), inches } \\ C_{L} & \text { Lift Coefficient } \\ C_{D} & \text { Drag Coefficient } \\ C_{Y} & \text { Sideforce Coefficient } \\ C_{\cdot} & \text { Rolling Moment Coefficient } \\ C_{m} & \text { Pitching Moment Coefficient } \\ C_{n} & \text { Yawing Moment Coefficient } \\ C_{n} & \text { Center of Gravity } \\ C_{P} & \text { Pressure Coefficient } \\ F S, x & \text { Fuselage Station (axial) Coordinate, inches } \\ \phi & \text { Forebody Polar Angle, degrees } \\ m & \text { Porous Surface Net Mass Flux, slug/sec } \\ M & \text { Mach Number } \\ R & \text { Computation Residual } \\ R_{o} & \text { Initial Computation Residual } \\ \text { WL, z } & \text { Water Line (vertical) Coordinate, inches }\end{array}$

\section{Aircraft Configuration}

The aircraft used in this investigation is based on fighter configurations developed under the Air Force Wright Lab "Aero Configuration/Weapons Fighter Technology" (ACWFT) program [24]. The goal of this program was to develop multi-mission fighter aircraft configurations with advanced technologies and performance characteristics capable of addressing postyear-2000 needs and threats. Special emphasis was placed on the design and performance of advanced aerodynamic control effectors for tailless or reducedtail concepts. Through a combination of wind-tunnel testing, performance analyses, and trade studies, a concept was selected for design refinement, resulting in the ACWFT 1204 configuration shown in Figure 4.

For the current study, a simplified aircraft configuration, dubbed "S1204", was developed by extracting salient features of the basic 1204 planform and outer mold line to form an analytically defined geometry (required for future studies involving reshaping). To reduce complexity, the 1204's cockpit and engine inlets were removed, and the exhaust nozzle was faired over. In addition, the 1204's NACA $6+$ series airfoil shape was replaced with a simpler $4 \%$ thick bi-convex airfoil. The resulting $\mathrm{S} 1204$ configuration is shown in Figure 5. As developed, the S1204 configuration preserves the relevant characteristics of the ACWFT 1204 and is a good generic testbed for advanced aerodynamic control effector concepts, making it well suited for the present investigation. Prior to the work discussed in this report. preliminary CFD analysis was conducted on the S1204 configuration at a variety of flow conditions to verify that its aerodynamic performance was consistent with the original ACWFT 1204.

\section{Computational Fluid Dynamics Simulation}

The NASA Langley unstructured computational fluid dynamics code "USM3D" [25] was used for NavierStokes analysis in this study. Within the tetrahedral cell-centered, finite volume flow solver, inviscid flux quantities are computed across each cell face using Roe's flux-difference splitting scheme. A novel reconstruction process is used for spatial discretization. based on an analytical formulation for computing gradients within tetrahedral cells. Solutions are advanced to a steady state condition using an implicit backward-Euler time-stepping scheme.

Within USM3D, turbulence closure is given by the Spalart-Allmaras one-equation model |26|. This model solves a single local transport equation for the turbulent viscosity. The turbulence model can be integrated down to the wall, or can be coupled with a turbulent boundary layer wall function to reduce the number of cells in the sublayer region of the boundary layer. The latter approach was used here.

\section{Computational Model}

The VGRID/GridTool software system $[27,28]$ was used to generate the unstructured grids for this study. VGRID uses an advancing-front method for generating Euler tetrahedral grids, and an advancing-layer method for thin-layer tetrahedral viscous grids required for Navier-Stokes analysis. In defining the computational domain, boundaries are represented by bi-linear surface patches that are constructed in GridTool based on userspecified geometries. Grid characteristics like cell spacing and stretching are also specified in GridTool b! the placement of cell "sources".

A surface mesh is generated in VGRID by triangulating each surface patch with a two-dimensional (2D) version of the advancing-front method. Triangulated surface patches then form the initial "front" for the generation of three-dimensional (3D) tetrahedral volume cells by the advancing-layer and advancing-front methods. Smooth variation of grid spacing is achieved by solving a Poisson equation on a cartesian background grid. using the GridTool-defined cell sources as inputs. 
Two grids were developed for this study: a semi-span grid suitable for longitudinal control analysis, and a full-span grid intended for lateral-directional control analysis. The semi-span grid with symmetry plane is shown in Figure 6, and the full-span surface mesh is shown in Figure 7 . In both cases, the computational domain extended roughly $10-14$ mean aerodynamic chord lengths from the aircraft $C G$ in all directions. The semi-span grid contained a total of 961481 tetrahedral cells and 168.390 nodes, and the full span grid contained twice that amount.

\section{Boundary Conditions}

Outer boundaries of the computational domain were treated as characteristic inflow/outflow surfaces with freestream conditions specified by Mach number, Reynolds Number, flow angle, and static temperature (the semi-span case used a reflection boundary condition at the symmetry plane). For comparison with the ACWFT database, low-speed high angle of attack conditions of $M=0.14$ and $\alpha=28^{\circ}$ were selected for analysis. Reynolds number was chosen to match the $1 \times 10^{6} / \mathrm{ft}-2 \times 10^{6} / \mathrm{ft}$ conditions of the various ACWFT wind tunnel tests.

Aircraft surfaces were treated as no-slip viscous boundaries. In cases involving the application of passive porosity control effectors, selected patches on the aircraft surface were treated as porous surfaces using the newly implemented porosity boundary condition in USM3D. The boundary condition is structured to allow any number of surface patches to communicate through a common plenum, with provisions for up to eight independent plenums. Specific details of this boundary condition will be given in a forthcoming paper $|29|$, so only a basic overview will be given here.

The USM3D porous boundary condition is an extension of the theory developed by Bush $|30|$ to model flow through a screen. Bush's original model was derived to pass information across a coterminous boundary separating an external flow and an internal plenum. In the revised approach used in USM3D, the Bush model was re-formulated as a surface boundary condition for the external flow, thus eliminating the need to grid and compute flow within a plenum. Conservation laws from steady, one-dimensional (ID), isentropic, and adiabatic gas dynamics are used to model flow through the porous surface, in conjunction with the assumption of a constant plenum pressure and the requirement of zero net mass flow through the porous surface. Part of the solution procedure involves a feedback iteration to update the plenum pressure and drive net mass flow to zero. Because of the $1 \mathrm{D}$ equations used in the boundary condition. only the surface porosity level is specified, not the actual porous hole geometry (circular holes of 0.020-0.050 inch diameter are typically used in wind tunnel and flight applications of passive porosity). Based on previous aerodynamic testing $11,17,22]$, a porosity level of $22 \%$ openness was used in this study.

The porous boundary condition has been validated by comparing computational results to experimental data obtained for a $\mathrm{GA}(\mathrm{W})-\mathrm{l}$ wing with leading-edge porosity $[1]$ and a 5 caliber, tangent-ogive forebody with circumferential porosity |22|. In both cases. computational results showed remarkable agreement with experimental force and pressure data |29|.

\section{Solution Procedure}

All solutions presented in this paper were obtained by running USM3D on the "Von Neumann" (ray (C-90 at NASA Ames, using 10 processors in multi-task mode. Typical cases needed 6000-8000 cycles for full convergence. Semi-span computations required about 175 megawords of memory and $60-85$ hours of computer time. Full span cases required double those amounts. During the computation, global CFL number ranged from 5 to approximately 30 .

Convergence was judged by several methods. The first involved tracking the solution residual until it dropped several orders of magnitude and leveled out. as shown in Figure 8(a). Integrated aerodynamic performance coefficients shown in Figure $8(b)$ were also used to verify convergence. Finally, in cases using the passive porosity boundary condition. average porous surface pressure. plenum pressure, and porous surface net mass flow were tracked. Typical histories of these parameters are shown in Figures 8(c) and 8(d).

\section{Baseline Results}

Results for the baseline $\$ 1204$ configuration at $M=0.14$ and $\alpha=28^{\circ}$ are given in Figures 9 and 10 . Figure 9 shows pressure coefficient $\left(C_{p}\right)$ contours over the upper and lower surface of the aircraft, and Figure 10 uses particle traces to illustrate the vortical flow field about the upper surface of the aircraft. These results are typical for a chined body at high angle of attack, and are in excellent agreement with the ACWFT flow visualization results obtained by McGrath. et al [31]. Two large vortices track along the upper surface of the forebody (red and green traces), and wing flow is characterized by a spanwise vortex originating from each leading-edge wing-body junction (blue (races). These vortical flows create significant low-pressure regions along the aircraft's upper surface, with a typical $C_{P}$ level of about -2 . As expected, the lower surface of the aircraft is dominated by high-pressure, with $C_{P}$ ranging from approximately 0 to $\mathrm{l}$. This combination of low- and high-pressure regions is ideal for the application of passive porosity. 


\section{Control Effector Design}

As discussed in the introduction, the basic premise of a passive porosity control effector system involves connecting low- and high-pressure regions of an aircraft to alter surface loading and generate maneuver control. This can be accomplished by allowing pressure to alter the surface loading directly, or by controlling a secondary flow (such as a vortex) which in turn alters surface loading. In general, connecting low-pressure upper surface regions with high-pressure lower surface regions tends to have the largest effect on flow in the low-pressure (upper surface) region. The high-pressure (lower surface) region typically shows little or no change, and basically acts as an unlimited high-pressure source $[1,17,20,22]$. Thus, the design of a passive porosity control effector system involves two basic steps: (1) identifying "target" regions on the aircraft's upper surface where the application of high-pressure will generate a desired force or moment, and (2) identifying corresponding high-pressure "source" regions on the aircraft"s lower surface. In the case of the S1204, where the entire lower surface contains high-pressure at high angle of attack, source regions can be chosen to simplify overall systems integration.

\section{Design Tool}

For a particular target region on an aircraft's upper surface, the effect of applying passive porosity will depend on several factors: the pressure distribution, the local surface geometry, and the location and moment arm relative to the aircraft $C G$. While the effect of these factors is easy to visualize in simple cases, it can pose a significant design challenge for complex geometries and arbitrary surface shapes. In order to streamline the design process, a simple computational tool was developed to help identify target areas for the application of passive porosity on an aircraft upper surface. Using USM3D solution and grid files as inputs, the tool surveys the surface geometry and configuration layout of low-pressure, upper-surface regions and calculates the potential forces and moments that would be generated if the local surface pressure coefficient were raised to a specified target level. For the present investigation, $C_{p}=0$ (freestream pressure) was deemed a suitable target value.

Results from the design tool are shown in Figure 11 (a) and 11 (b), for nose-down pitch and yaw, respectively (moments referenced to the stability axes). In each plot, potential increments in moment are plotted per unit area over the aircraft upper surface. It is important to note that results from the design tool do not indicate the direct effects of applying passive porosity; rather, they indicate the effect of raising local pressure on the upper surface. Passive porosity is simply one method of accomplishing this, either by direct loading or through the control of secondary flows.
Results in Figure Il(a) indicate that target areas for nose-down pitch control are the forebody and leading edge wing-body junction regions (forward of the aircraft CG). Low-pressure in these regions is due to vortical flows, and control of these flows is key to obtaining nose-down pitch. Potential yaw control increments in Figure 11(b) show a similar result. and indicate that "asymmetric" control of the vortical flows is required for yaw generation. At the $\alpha=28^{\circ}$ condition, stability-axes yaw depends heavily on both yaw and roll about the body axes. Raising pressure on one side of the forebody will effect body-axes yaw. while raising pressure on the upper surface of one wing will effect body-axes roll. Combined, these moments will contribute to yaw in the stability-axes.

\section{Control Effector Configurations}

Based on the design analysis, ten passive porosity configurations were developed to effect pitch and yaw control. Target regions on the aircraft upper surface were mated with corresponding source regions on the lower surface (i.e., lower surface porosity was a direct projection of upper surface porosity). From a systems standpoint, this approach would minimize the amount of complexity involved in connecting upper and lower surface regions by a common plenum, making it a good starting point for design.

Five proposed pitch control configurations are shown in the top row of Figure 12. Configurations P1 and P2 apply porosity to the forebody region, starting at the nose and going back to FS $=147$ (covering $33 \%$ of the forebody area) and $\mathrm{FS}=298$ (covering 100\%). respectively. Configuration $\mathrm{P} 3$ applies porosity to the leading-edge wing-body junction region, forward of the aircraft $C G$ at $F S=365$. Configurations $P 4$ and $P 5$ are combinations of the P1, P2, and P3 designs.

Five proposed yaw control configurations are shown in the bottom row of Figure 12. Configurations $\mathrm{Y} 1$ and Y2 are asymmetric counterparts of the $P 1$ and $P 2$ configurations, covering $17 \%$ and $50 \%$ of the total forebody area, respectively. The Y3 configuration is similar to the P3 configuration, except that porosity extends further back to $50 \%$ chord, and is applied to the left wing only. Finally, the $\mathrm{Y} 4$ and $\mathrm{Y} 5$ configurations are combinations of the Y1, Y2, and Y3 designs.

A single plenum was used to connect upper and lower surface porosity in the $\mathrm{Pl}, \mathrm{P} 2, \mathrm{Y} 1, \mathrm{Y} 2$, and $\mathrm{Y}$ ? configurations. Two separate plenums were used for the P3 case, one for each wing-body junction region. Three separate plenums were used in the P4 and P.5 cases, one for the forebody region, and one for each wing-body junction region. Similarly, two separatc plenums were used in the asymmetric Y4 and Y5 cases, one for the forebody, and one for the left wing. 


\section{Longitudinal Control}

Results for the P1 configuration are shown in Figures 13-16. Comparing the $C_{p}$ contours in Figure 13 with those of the baseline configuration (Figure 9), it is obvious that the application of passive porosity resulted in a large overall increase in local pressure on the forward upper surface of the forebody, but pressure on the lower surface was virtually unchanged (as expected). Figure 14 gives a plot of surface $C_{P}$ versus polar angle around the forebody at $F S=100$, for the baseline and PI configurations. At this location, the upper surface pressure in the $\mathrm{Pl}$ configuration is nearly constant at $C_{P} \approx-0.65$, leveling out the suction peaks of the baseline configuration.

Downstream of the porous region, the upper surface pressure distribution is very similar to the baseline case. Looking at the particle traces depicted in Figure 15, it appears that passive porosity delayed onset of the forebody vortices: instead of rolling up at the nose, the vortices formed downstream of the porous region, as indicated by the red particle traces. Crossflow streamlines at $\mathrm{FS}=100$ are shown in Figure 16(a) and 16(b). for the baseline and P1 configurations, respectively. Based on the information shown here, it would seem that passive porosity prevented windward flow from rolling into vortices as it separated across the chine. This is likely a direct result of mass transfer out of the upper porous surface, which blunted the effect of the chine, filled in the wake on the leeward side of the forebody, and deterred the formation of secondary flows.

Results for the P2 configuration are shown in Figures 17-20. In this case, the application of passive porosity provided a notable increase in upper surface pressure on the entire forebody, and also increased pressure in the leading-edge wing-body junction region. As a result. formation of the forebody and wing body junction vortices was greatly inhibited. Instead of the distinct vortex families seen in the baseline and PI cases, particle traces for the $\mathrm{P} 2$ configuration roll up into a larger, less organized vortex system on each side of the aircraft (see Figure 18).

An upper surface $C_{p}$ plot for the $\mathrm{P} 3$ configuration, with passive porosity applied to the leading-edge wing region forward of the $\mathrm{CG}$, is given in Figure 21 . These results show that the application of passive porosity increased pressure and smoothed out pressure gradients in the leading-edge wing-body junction region. This is confirmed by the $C_{p}$ plot in Figure 22 (taken at the spanwise location $B L=-100$ ), which shows that the wing's suction peak was leveled off to a near constant value over much of the upper surface, while the lower surface pressure was largely unaffected. Based on the vortical flow field depicted in Figure 23, it appears that passive porosity completely eliminated the wing-body junction vortices seen in the previous configurations. Here, blue particle traces originating from the wingbody junction region roll into the forebody vortices. which are better defined and more coherent than the baseline case.

Results for the P4 and P5 configurations are shown in Figures 24-27. As expected. these configurations incorporate flow features and characteristics from the basic P1-P3 porosity layouts. The P5 configuration shows the most striking results: by combining full forebody porosity from P2 with wing porosity from P3. major pressure gradients on the upper surface of the $P 5$ configuration appear to be completely blended out. Accordingly, particle traces for the P5 configuration in Figure 27 show a lack of the strong vortical flows present in earlier cases, with only minor rollup over the aft portion of the aircraft.

\section{Pitch Increments}

Nose-down pitch control effectiveness for the five passive porosity configurations is summarized in Figure 28 , along with data for selected ACWFT pitch control effectors $|24|$. The passive porosity control effectors provided nose-down pitch increments ranging from $\Delta C_{m}=-0.089$ for the $P 1$ configuration to $\Delta C_{m}=-0.31$ for the P5 configuration, comparing favorably to the range of ACWFT devices and conventional controls. Both the P2 and P5 configurations provided enough nose-down pitch increment to reach "absolute" nosedown control, countering the configuration's inherent nose-up pitching moment at the $\mathbf{M}=0.14, \alpha=28^{\circ}$ condition. With increments of $\Delta C_{\mathrm{m}}=-0.243$ and $\Delta C_{m}=-0.31$. respectively, the $P 2$ and $P 5$ configurations roughly equaled or exceeded the $\Delta \mathrm{C}_{\mathrm{m}}=-0.25$ provided by the conventional ACWFT elevons deflected to $60^{\circ}$.

\section{Associated Lift Increments}

Lift increments for the P1-P5 configurations are shown in Figure 29, along with estimates made from the ACWFT data $|24|$. Like the ACWFT forebody derices. the porous configurations generate nose down pitch by reducing local lift on the aircraft forebody, which reduces overall lift in each case. This is in contrast to conventional aft-mounted pitch effectors. which increase overall lift. Relative to the baseline configuration's $C_{L}=1.748$, the P1, P3, and P4 configurations reduced lift by $6-13 \%$. These levels compare favorably to the ACWFT forebody devices. which reduced lift by $4-17 \%$ for similar nose-down pitch levels. The P2 and P5 configurations reduced lift by larger amounts $-17 \%$ and $26 \%$, respectively - but these reductions are commensurate with the larger pitch increments provided by these configurations. 


\section{Pitch Control Trends}

Using the baseline and porous results obtained here as guidance, one can construct the trends given in Figure 30, which show that the passive porosity pitch system provides control effect that varies linearly with porous forebody area. These results indicate that a simple means of controlling the open porous area on the forebody (such as the sliding plates or louvers shown in Figure 3) would yield a passive porosity effector system with linear, variable pitch control.

\section{Lateral-Directional Control}

Results for the $\mathrm{Y} 1$ and $\mathrm{Y} 2$ yaw control configurations are presented in Figures 31-36. As asymmetric counterparts of the $P 1$ and $P 2$ configurations, the pressure and flow field results seen here are consistent with earlier discussion. The partial porosity of the Y I configuration is seen to raise pressure on the forward-left upper surface region of the forebody and delay the onset of the vortex on that side, while the full porosity of the $\mathrm{Y} 2$ configuration raised pressure on the entire left side of the forebody upper surface and nearly eliminated the vortex on that side. $C_{P}$ plots for the $Y 2$ configuration at $\mathrm{FS}=100$ and 220 (Figures 35 and 36) more clearly show the asymmetric effect of passive porosity. While pressure on the left upper surface region of the forebody $\left(180-270^{\circ}\right)$ changed considerably from the baseline case, the remainder of the forebody surface pressure was unaffected to a large extent. This demonstrates the capability of passive porosity to act as a localized control effector.

Results for the Y3 configuration are shown in Figures 37 and 38, and are in line with previous results seen for the similar P3 configuration. The application of passive porosity to the forward half of the left wing is seen to have a notable effect on the upper surface pressure distribution shown in Figure 37. Porosity smoothed out pressure gradients in the wing-body junction area, eliminated the wing-body junction vortex, and raised the overall wing upper surface pressure. As shown in Figure 39, the wing suction peak was reduced and upper surface pressure was leveled off to a near constant value of $C_{P} \approx-0.9$ at the $B L=-100$ location.

Upper surface $C_{P}$ plots and particle traces for the composite $\mathrm{Y} 4$ and $\mathrm{Y} 5$ configurations are given in Figures 40-43. These results are amalgamations of earlier results seen for the Y1-Y3 configurations, combining the various elements from each case. Not surprisingly, the $\mathrm{Y} 5$ configuration appears to give the best results, by combining full forebody porosity (Y2) with wing porosity (Y3) on the left side of the aircraft. Particle traces in Figure 43 show only weak signs of vortical flow along the left side of the aircraft, with slow rollup aft of the wing.

\section{Yaw Increments}

Yaw control effectiveness for the Y1-Y5 configurations is summarized in Figure 44, along with ACWFT control effector and historical data [24]. The passive porosity control effectors provided yaw increments ranging from $\Delta C_{n}=0.012$ to 0.051 , compared with the levels of 0.018 to 0.020 for conventional rudder controls on the F-15 and F/A-18, and 0.011 to 0.025 for the various ACWFT devices. While the YI and Y3 configurations provided yaw increments in line with the ACWFT devices and conventional rudders, the Y2, Y4. and $Y 5$ configurations performed significantly better, generating 2-5 times more yaw than the other effectors.

\section{Associated Forces and Moments}

Adverse roll increments are presented in Figure 45 , which shows that roll levels generated by the Y1-Y.5 porous yaw control effectors are in line with other ACWFT devices [24], and well within the $\Delta C_{1}=0.06$ level of corrective roll authority available from the ACWFT aileron system. The porous yaw control effectors also induced nose-down pitch and lift increments, summarized in the table below.

Table 1: Assoc. Pitch \& Lift Increments, YI-Y5 Configs.

\begin{tabular}{|c|c|c|c|c|c|}
\hline & Y1 & Y2 & Y3 & Y4 & Y5 \\
\hline$\Delta \mathbf{C}_{\mathbf{m}}$ & -0.035 & -0.121 & -0.060 & -0.098 & -0.180 \\
\hline$\Delta \mathbf{C}_{\mathbf{L}}$ & -0.030 & -0.114 & -0.149 & -0.179 & -0.226 \\
\hline
\end{tabular}

Ideally, the generation of yaw should be accomplished with as little net sideforce as possible. Figure 46 shows sideforce increments generated by the Y1-Y5 porous configurations compared to estimates from $\mathrm{ACWFI}$ data and typical historical levels [24]. Increments of $\Delta C_{Y}=0.029-0.039$ for the $Y 1, Y 3$, and $Y 4$ cases fall below the sideforce levels of the ACWFT devices and conventional rudders for equal or greater yau increments, indicating that the porous configurations are more effective at producing yaw. The Y2 and Y5 configurations produced larger sideforce increments, of 0.076 and 0.105 respectively, but these levels are consistent with the larger amounts of yaw produced by these control effectors. In reality, the $\mathrm{Y} 2$ and $\mathrm{Y} 5$ configurations produce about the same amount of sideforce for a given yaw as the other porous cases.

\section{Yaw Control Trends}

As in the previous discussion of pitch control effectors, the baseline and Y1-Y5 data were used to construcl control trends, shown in Figure 47. Like the pitch case. the passive porosity system is seen to provide yau control increments that vary linearly with porous forebody area. 


\section{Concluding Remarks}

Advanced aerodynamic design and analysis of passive porosity control effectors has been conducted in this investigation. Using a generic tailless fighter aircraft concept, Navier-Stokes (FD and aerodynamic design tools were applied to develop control effectors capable of generating longitudinal and lateral-directional control for low-speed high angle of attack conditions. Specific conclusions and comments are as follows:

1. For longitudinal (pitch) control, the P1-P5 passive porosity effectors provided nose-down pitch increments that were competitive with ACWFT devices and conventional controls. Two of the porous configurations actually produced large enough pitch increments to effect absolute nose-down control at the high-alpha condition, equaling or exceeding the control authority provided by conventional elevons. The pitch control came with reductions in overall lift ranging from 6 to $26 \%$. Passive porosity pitch control effectors were seen to provide control increments that were a linear function of porous forebody area.

2. In terms of lateral-directional control, the $\mathrm{Y} 1$ and $\mathrm{Y} 2$ porous control effector configurations generated yaw increments in the range of conventional rudder controls and ACWFT devices. The remaining three porous configurations had yaw increments that were 2-5 times greater than the other effectors. All of the porous configurations generated acceptable levels of adverse roll, and provided less sideforce for a given yaw than conventional rudders and ACWFT devices. Yaw control increments were also seen to form a linear trend with porous forebody area.

3. This work demonstrates the tremendous potential of passive porosity to yield simple control effector systems that have no external moving parts and will preserve an aircraft's fixed outer moldline. Based on the linear behavior observed in this study for low-speed high-alpha conditions, a fully implemented variable geometry passive porosity system (using simple sliding plates or louvers to control porous area) would be able to provide continuous increments in nose-down pitch control ranging from $\Delta C_{\mathrm{m}}=0$ to -0.3 , and yaw control increments ranging from $\Delta C_{n}=-0.05$ to 0.05 .

4. Though the current study focused on low-speed high-alpha conditions, the passive porosity control effector concept applies equally well to other flight regimes, including low-lift conditions or other cases where pressure differentials on an aircraft surface are small. In these instances, planform layout and surface contouring may be used to modify local pressure gradients, and become a key part of control effector design. As such, development of full-envelope passive porosity control effectors may require new planforms and surface shapes.

\section{Acknowledgements}

The authors would like to thank Neal Frink. Paresh Parikh, and Shahyar Pirzadeh for their help and support with USM3D, VGRID, and ViGPlot. all part of the Tetrahedral Unstructured Software System "TetrUSS" developed at NASA langley.

\section{References}

1. Wood, R.M. and Bauer, S.X.S. "Advanced Aerodynamic Control Effectors". SAE Paper, 1999-01-5619. October 1999.

2. Scott, M., Montgomery, R., and Weston, R. "Subsonic Maneuvering Efectiveness of High Performance Aircraft which Fmploy Quasi-Static Shape Change Devices“. SPIE 3326-24, 1998.

3. Dorsett, K.M. and Mehl, D.R. "Innovative ('ontrol Effectors (ICE)". Wright Laboratory Report WI,-TR-96-3043, January 1996.

4. McGowan. A.R., Horta, L.G., Harrison. J.S., and Raney, D.L. "Research Activities Within NASA's Morphing Program" NATO-RTO Workshop on Structural Aspects of Flexible Aircraft Control. Ottawa, Canada, October 18-21, 1999.

5. Kudva, J.N., Martin. C.A., Scherer, L.B., Jardine, A.P., McGowan, A.R., Lake, R.C.. Sendeckyj, G.P., and Sanders, B.P. "Overview of the DARPA/AFRL/NASA Smart Wing Program". SPIE Paper 3674-26. March 1999.

6. Pack, L.G., and Joslin. R.D. "Overview of Active Flow Control at NASA Langley Research Center". SPIE Paper 3326-24, 1998.

7. Donovan, J.F., Kral, I.D., and Cary, A.W. "Active Flow Control Applied to an Airfoil". AIAA 98-0210, January 1998.

8. Seifert. A. and Pack. L.G. "Oscillatory Control of Seperation at High Reynolds Numbers". AIAA 98-0214. January 1998.

9. Nae, C. "Synthetic Jet Influence on a NACA 0012 Airfoil at High Angles of Attack". AIAA 98-4.523.

10. I achowicz. J.T., Yao, C., and Wlezien. R.W. "Scaling of an Oscillatory Flow-Control Device". AIAA 98-0330. January 1998.

11. Ho. C.M. and Tai, Y.C. "Review: MEMS and its Applications for Flow Control". Journal of Fluids Engineering $118(.3), 1996$. 
12. Joslin, R., Horta, L., and Chen, F. "Transitioning Active Flow Control to Applications".

AIAA 99-3575, June 1999.

13. Kral, L., Donovan, J., Cain, B., and Cary, A. Numerical Simulation of Synthetic Jet Actuators". AIAA 97-1824, 1997.

14. Raghunathan, S. "Passive Control of Shock Boundary Layer Interaction". Progress in Aerospace Sciences, Volume 25, 1988.

15. Nagamatsu, H.T., Trilling, T.W., and Bossard, J.A. "Passive Drag Reduction on a Complete NACA 0012 Airfoil at Transonic Mach Numbers". AIAA 87-1263, June 1987.

16. McCormick, D.C. "Shock - Boundary Layer Interaction Control with Low-Profile Vortex Generators and Passive Cavity". AIAA 92-0064, January 1992.

17. Bauer, S.X.S., and Hernandez, G. "Reduction of Cross-Flow Shock-Induced Separation with a Porous Cavity at Supersonic Speeds". AIAA 88-2567, June 1988.

18. Raghunathan, S. "Passive Shockwave Boundary Layer Control Experiments on a Circular Arc Model". AIAA 86-0285, January 1986.

19. Nagamatsu, H.T., Brower, W.B., Bahi, L., and Marble, S.K. "Investigation of Passive Shock Wave / Boundary Layer Control for Transonic Airfoil Drag Reduction“. First Annual report for NASA Grant NSG 1624, 10/1/79 to 9/30/80.

20. Wood, R. M.; Banks, D. W.; and Bauer, S. X. S. Assessment of Passive Porosity with Free and Fixed Separation on a Tangent Ogive Forebody. AIAA 92-4494, 1992.

21. Wilcox, F. J. "Experimental Investigation of the Effects of a Porous Floor on Cavity Flow Fields at Supersonic Speeds". NASA TP 3032, 1990.

22. Bauer, S. X. S. and Hemsch, M.J. "Alleviation of Sideforce on Tangent-Ogive Forebodies Using Passive Porosity". Journal of Aircraft, Vol 31, No 2, p354-361. March-April 1994.

23. NASA Langley Research Center, Office of External Affairs. "NASA Contributions to F/A18E/F: Centerpiece of US Navy's Carrier-Based Fighter/Attack Fleet Updated with NASA Aeronautics Technology". FS-1999-08-46-LaRC, August 1999.
24. O'Neil, P.J., Krekeler, G.C., Billman, G.M., and Creasman, F. "Aero Configuration / Weapons Fighter Technology (ACWFT) - Summary Technical Report”. Wright Labs WL-TR-95-3002. December 1994.

25. Frink N. "Tetrahedral Unstructured Navier-Stokes Method for Turbulent Flows". AIAA Journal, Vol. 36. No. 11, pp 1975-1982. November 1998.

26. Spalart, P.R., and Allmaras, S.R. "A One-Equation Turbulence Model for Aerodynamic Flows“. AIAA 92-0439. January 1992.

27. Pirzadeh, S. "Progress Toward a User-Oriented Unstructured Viscous Grid Generator". AIAA 96-003I, January 1996

28. Samareh, J. "GridTool: A Surface Modeling and Grid Generation Tool". Proceedings of the Workshop on Surface Modeling. Grid Generation, and Related Issues in CFD Solutions. NASA CP-3291, May 1995.

29. N. Frink, D. Bonhaus, V. Vatsa, S. Bauer, E. Nielsen, and A. Tinetti. "A Boundary Condition for Simulation of Flow over Porous Surfaces". Extended abstract for the 19th AIAA Applied Aerodynamics Conference to be held in Anaheim. California, June 11-14, 2001.

30. Bush, R. H. “Engine Face and Screen Loss Models for CFD Applications”. AlAA 97-2076. June 1997.

31. B. McGrath, D. Neuhart, G. Gatlin, and P. O'Neil. "Low-speed Longitudinal Aerodynamic Characteristics of a Flat-Plate Planform Model of an Advanced Fighter Configuration". NASA TM-109045, March 1994. 


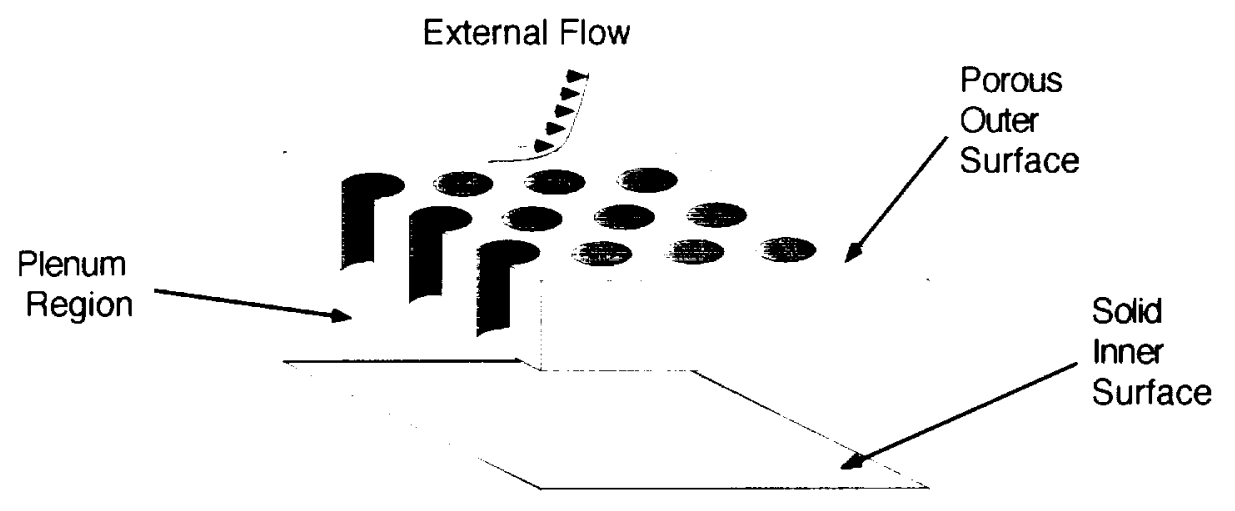

Figure 1: Passive Porosity Concept

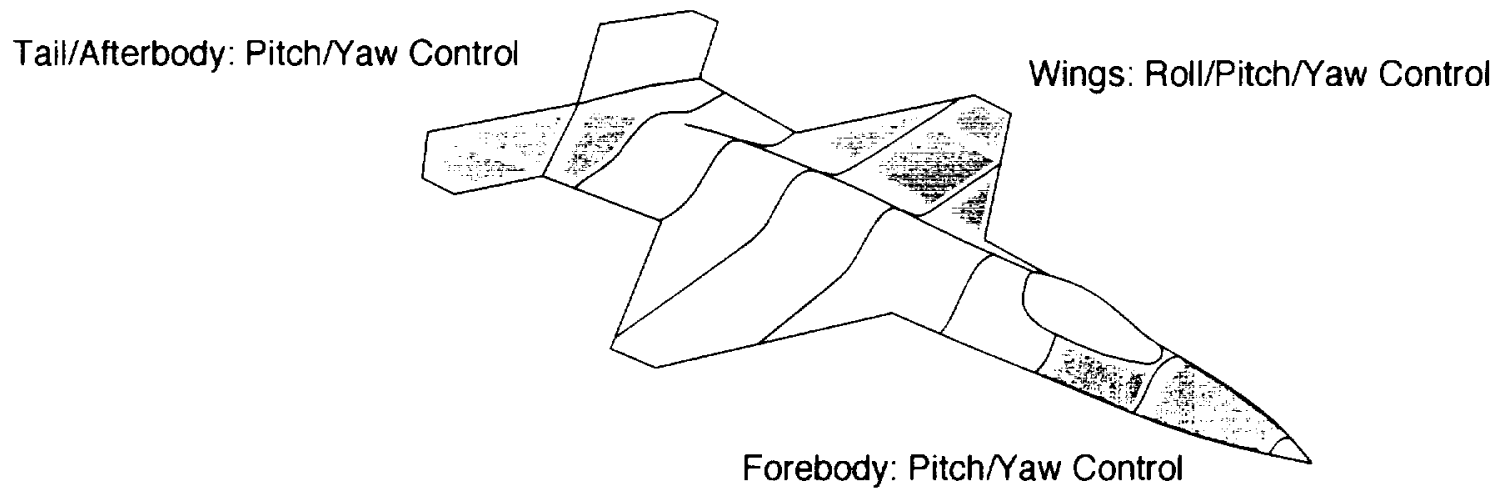

Figure 2: Passive Porosity Aerodynamic Control Effector Concept

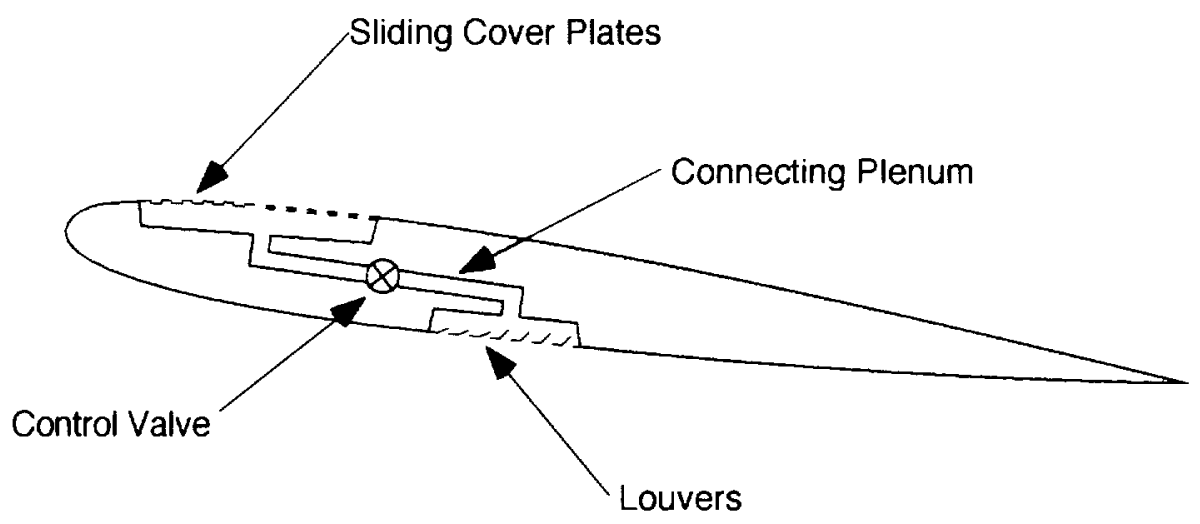

Figure 3: Passive Porosity Control Effector Schematic 


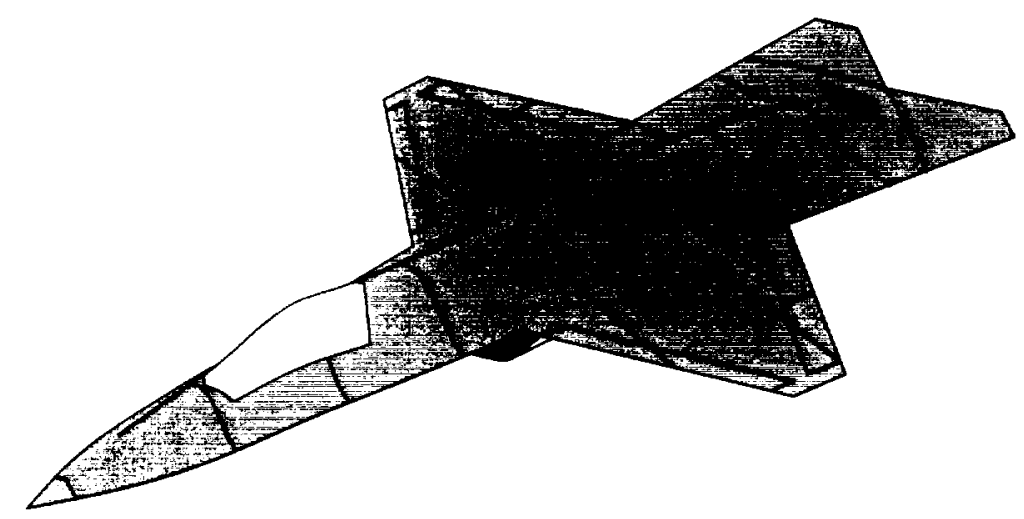

Figure 4: ACWFT 1204 Configuration

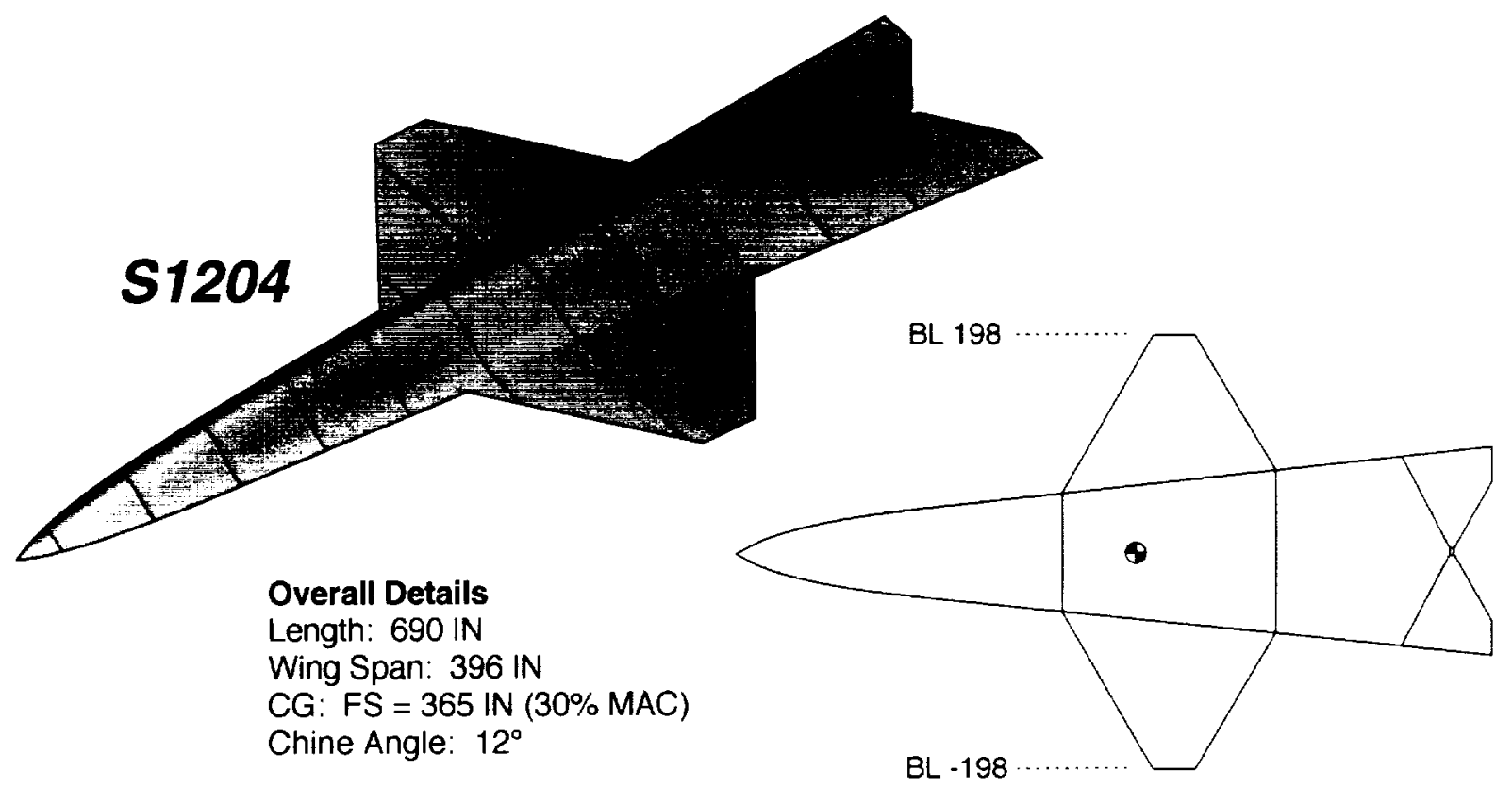

WL 125

WL 93

WL 58

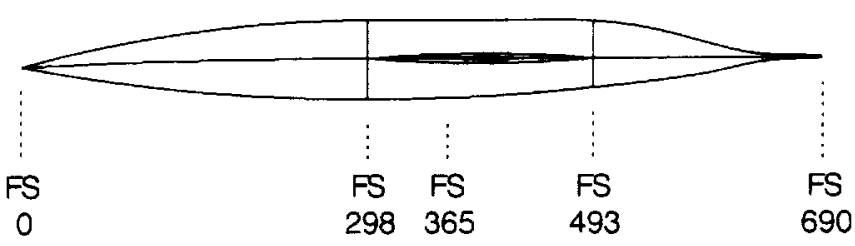

Wing Details

4\% t/c Bi-Convex Airfoil

Area: $425 \mathrm{sq} \mathrm{ft}$

LE/TE Sweep: $30^{\circ}$

MAC: 182 IN

Figure 5: Simplified S1204 Configuration 


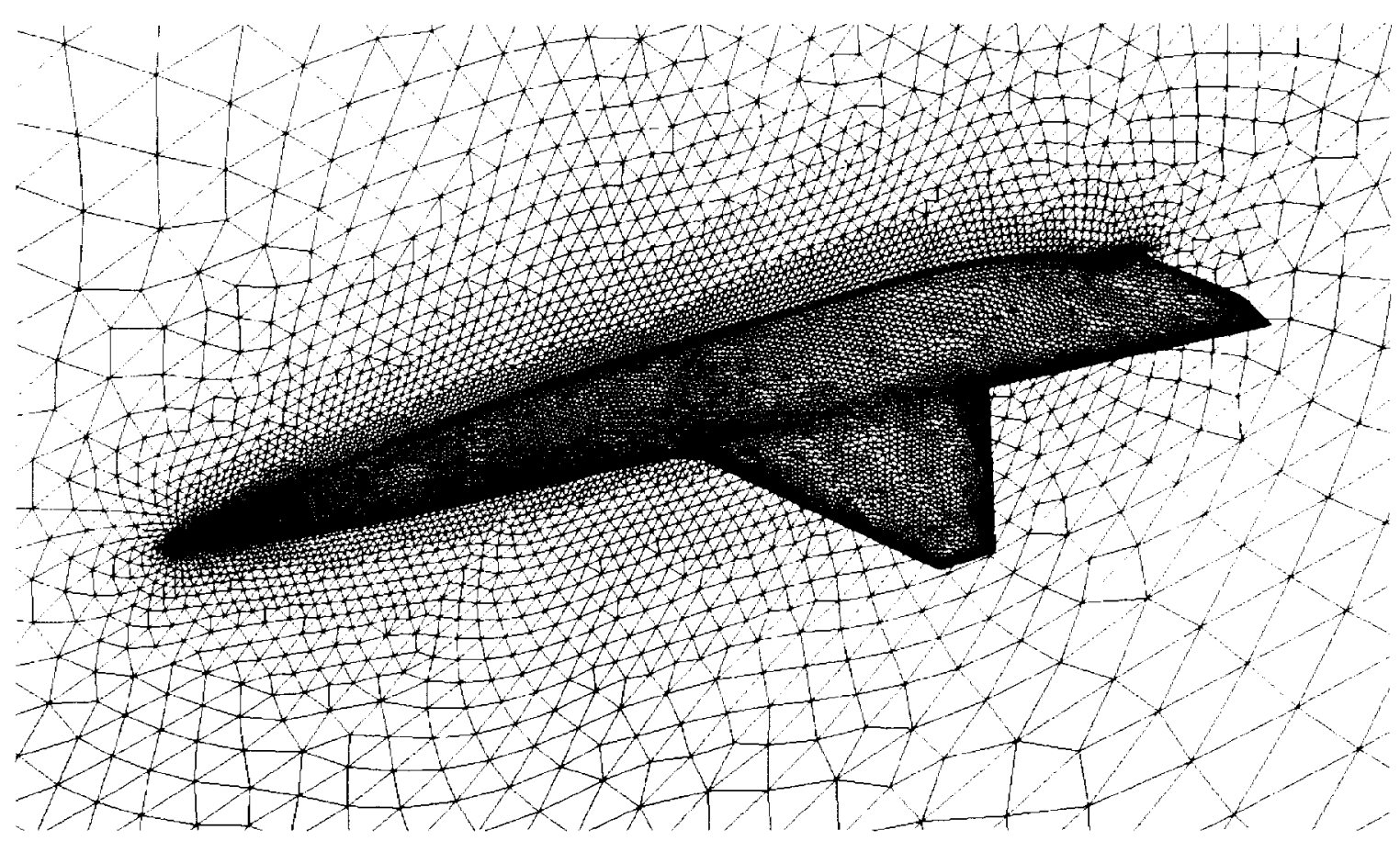

Figure 6: Semi-span S1204 Computational Grid

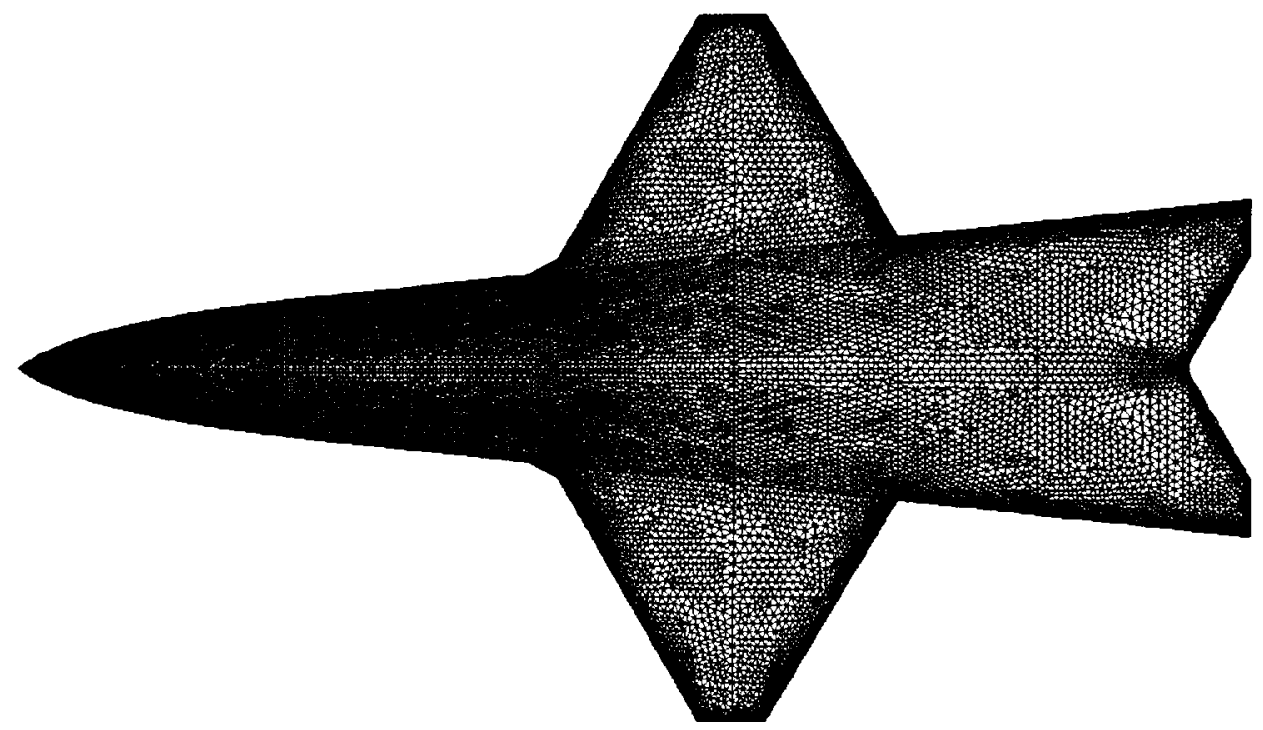

Figure 7: Full-span S1204 Surface Mesh 


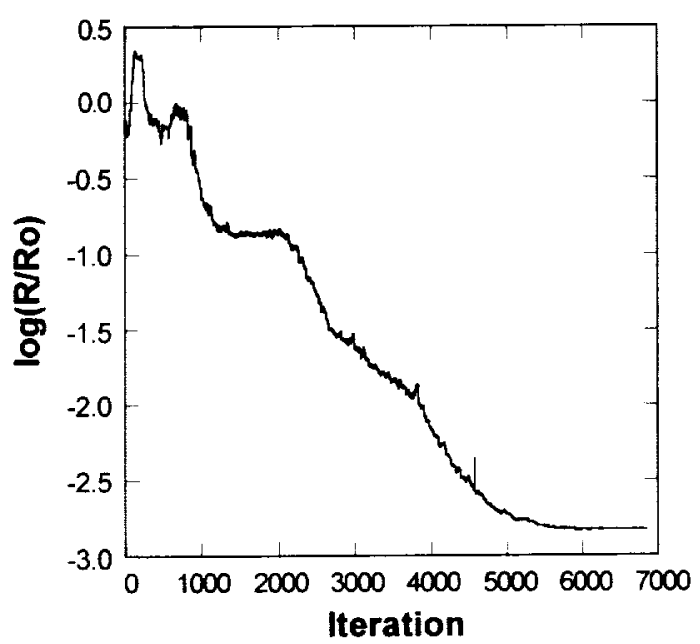

8(a) Residual

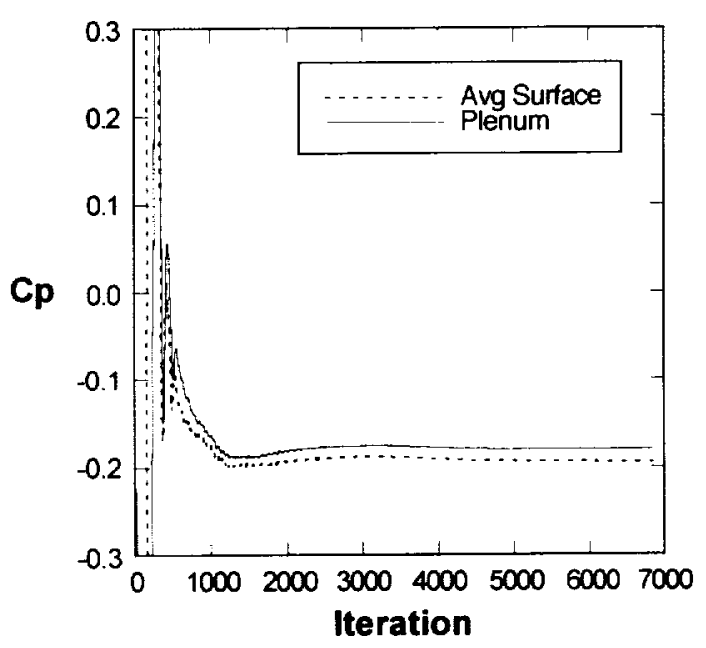

8(c) Porous BC Pressures

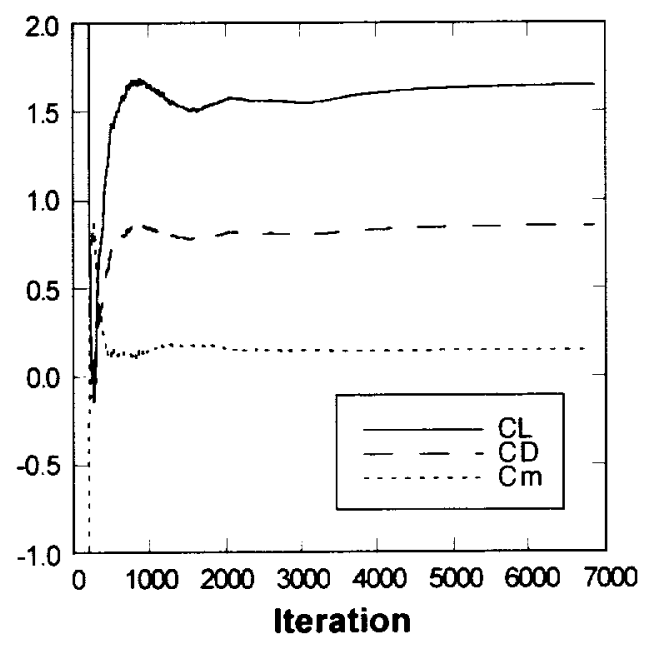

8(b) Forces and Moments

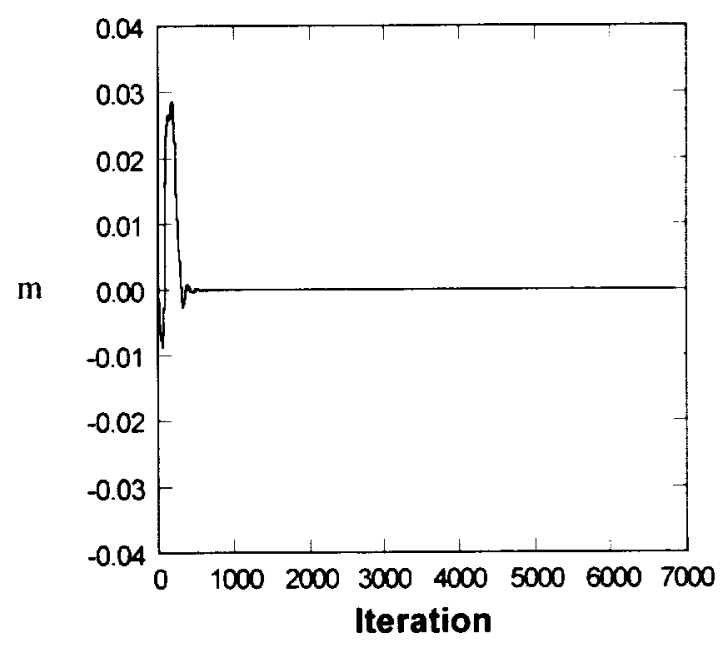

8(d) Porous Surface Net Mass Flow

Figure 8: Typical Convergence History 


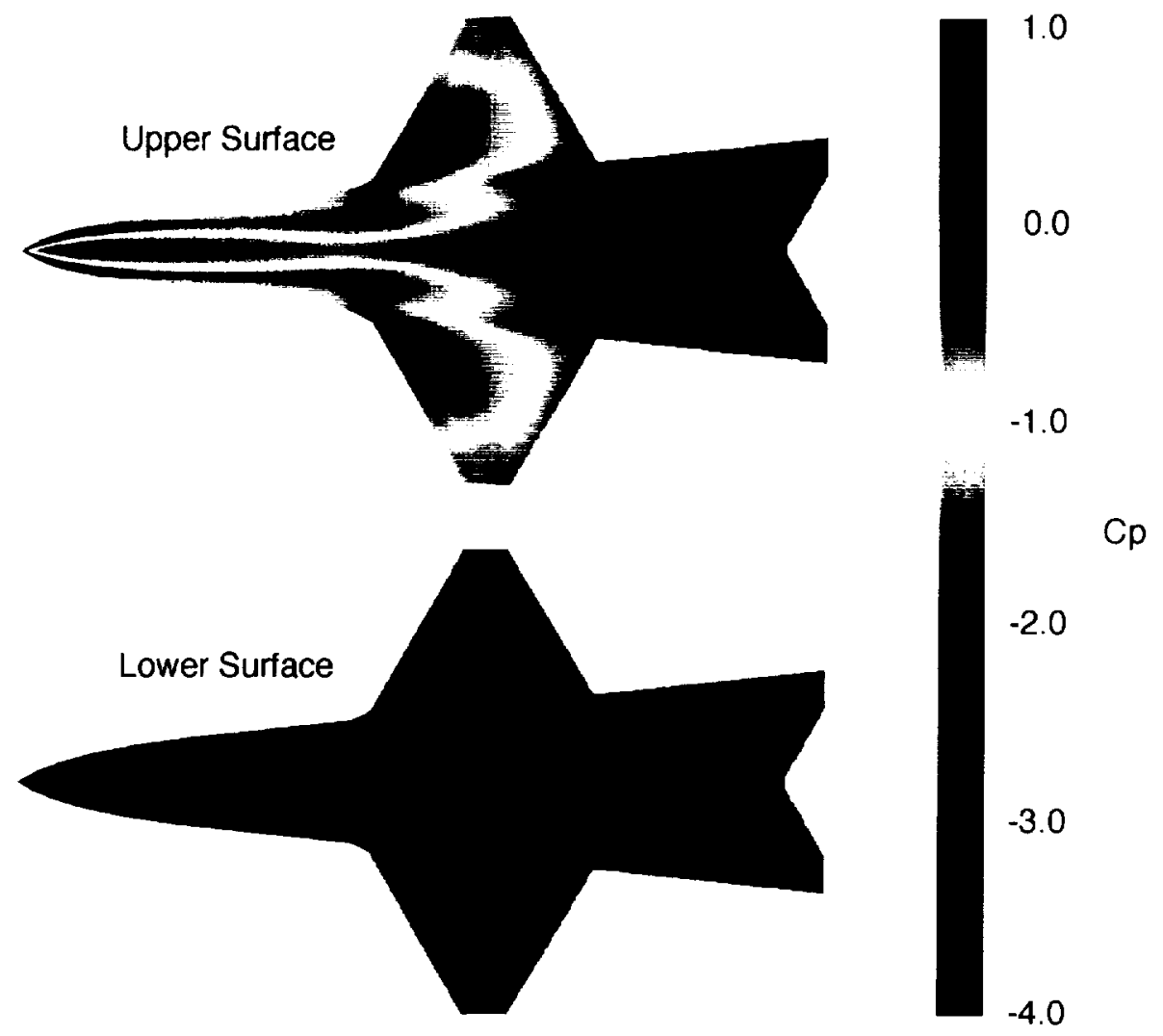

Figure 9: Upper and Lower Surface Pressure Coefficient-Baseline Configuration $\left(M=0.14,\left(x=28^{\circ}\right)\right.$

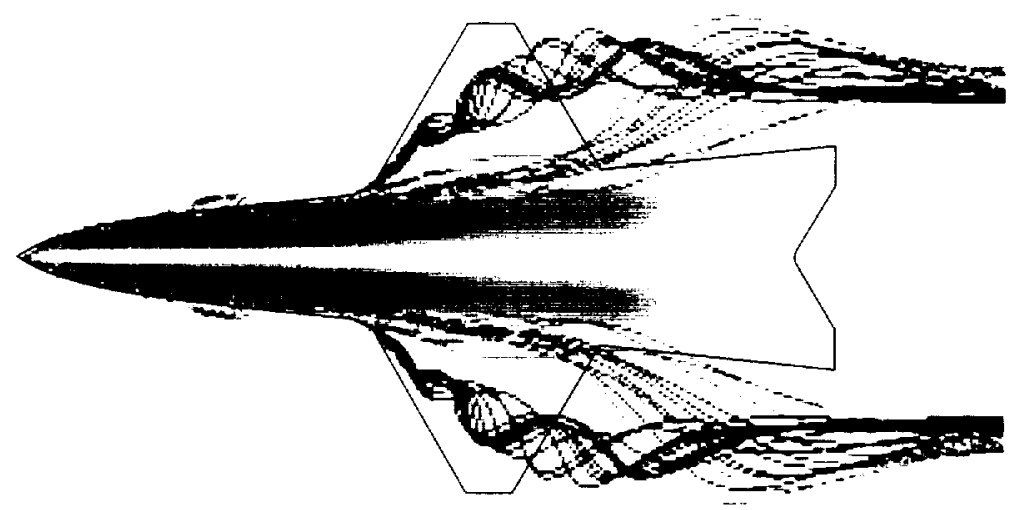

Figure 10: Upper Surface Vortex Flow - Baseline Configuration $\left(M=0.14, \alpha=28^{\circ}\right)$ 


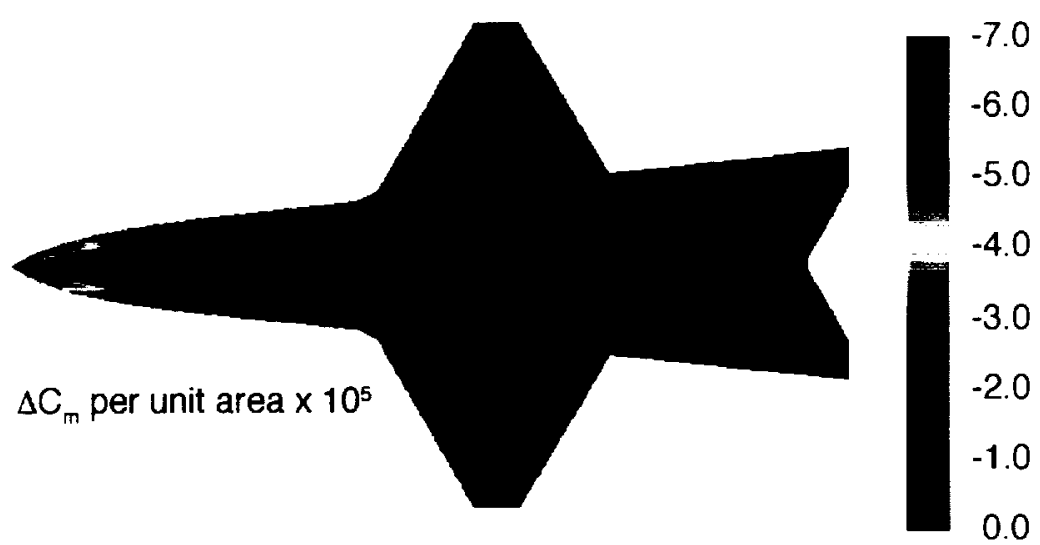

1 I(a) Nose-down Pitching Moment

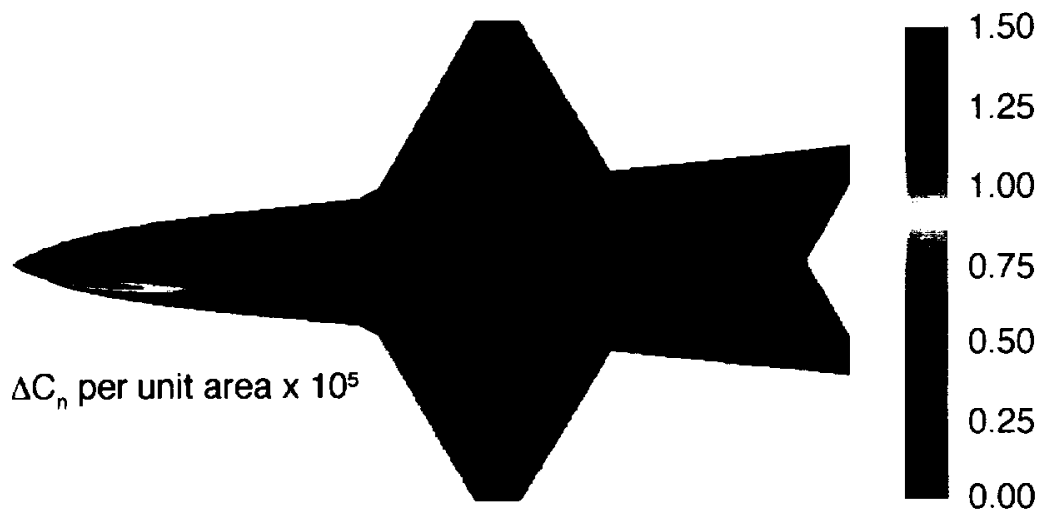

11(b) Yawing Moment

Figure 11: Target Areas for Passive Porosity - Baseline S1204 Upper Surface
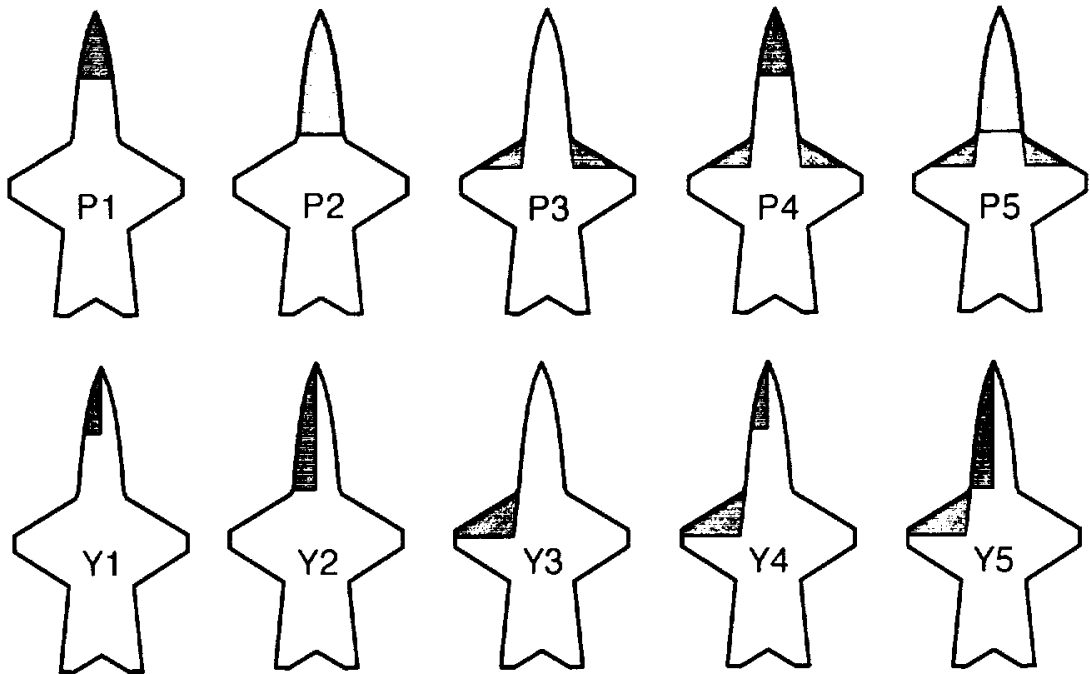

Figure 12: Passive Porosity Control Effector Configurations. P1-P5 for Nose-down Pitch Control, Y 1-Y 5 for Yaw Control.

Shaded regions indicate upper and lower surface porosity. 

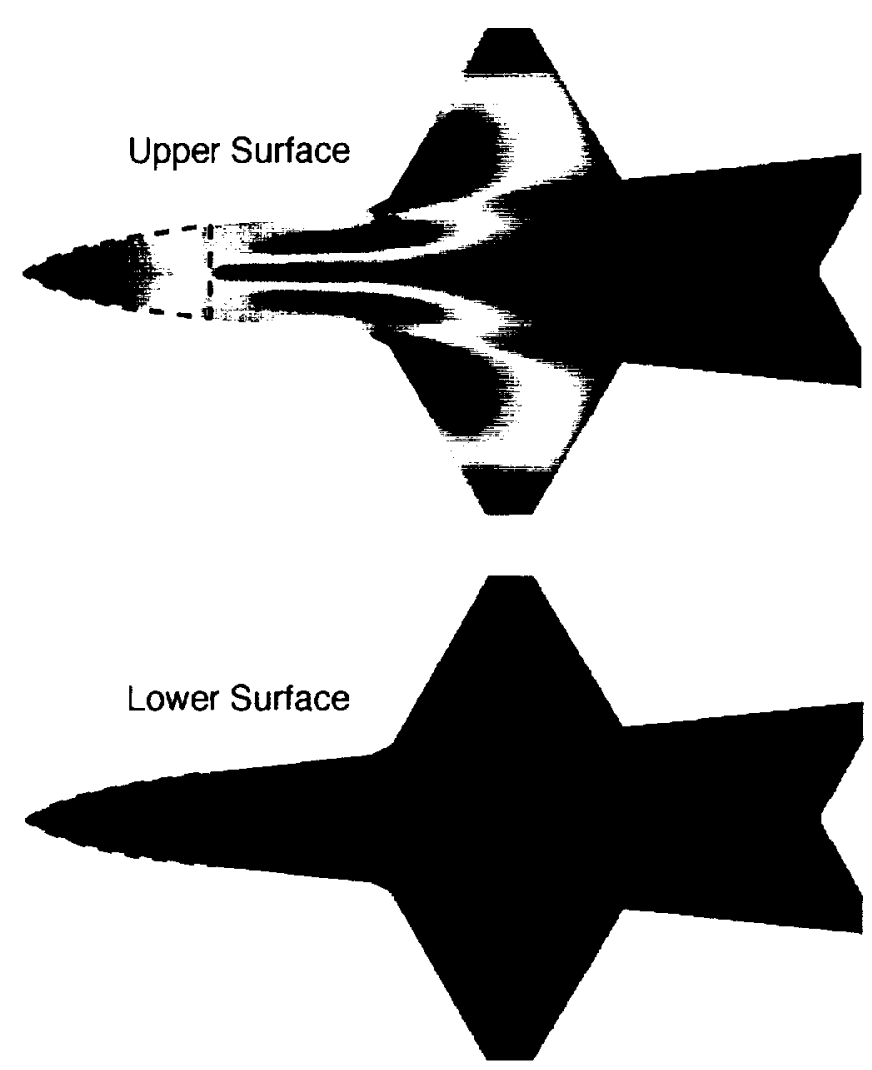

Figure 13: Upper and Lower Surface Pressure Coefficient - PI Pitch Control Configuration Dashed lines indicate porous regions.

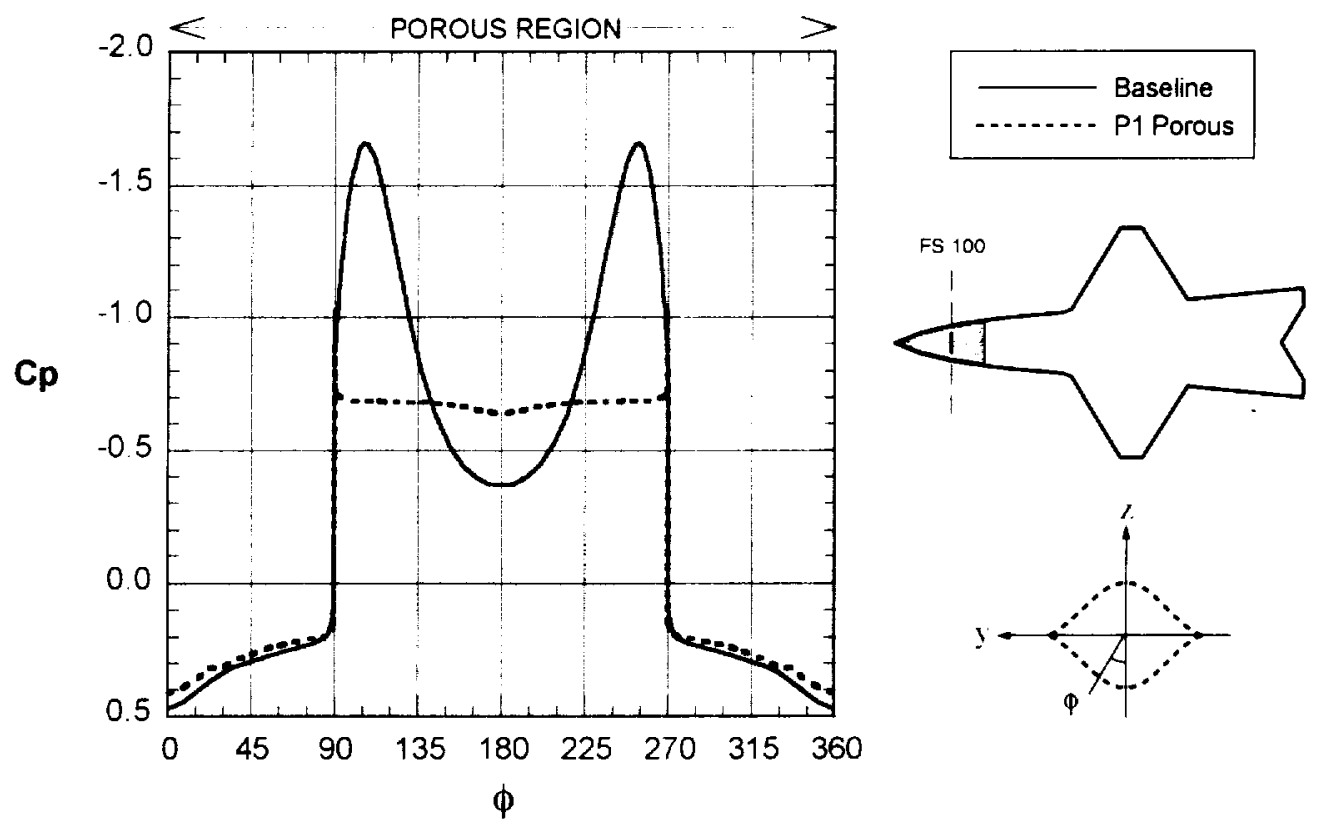

Figure 14: Comparison of Forebody Surface Pressure Coefficient at FS $=100$ Buseline and PI Pitch Control Configuration 


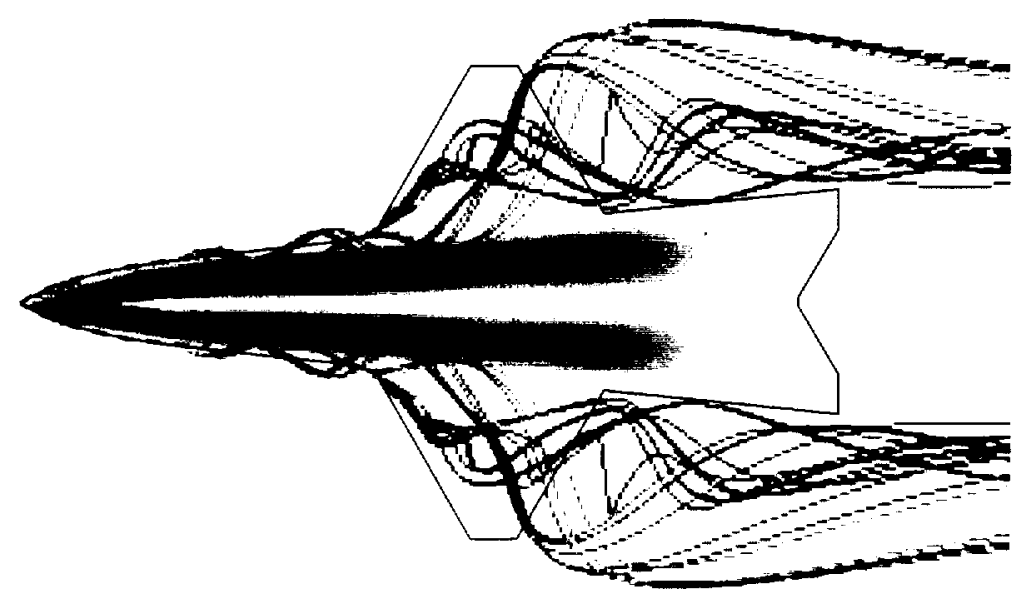

Figure 15: Upper Surface Vortex Flow - P1 Pitch Control Configuration Dashed lines indicate porous regions.

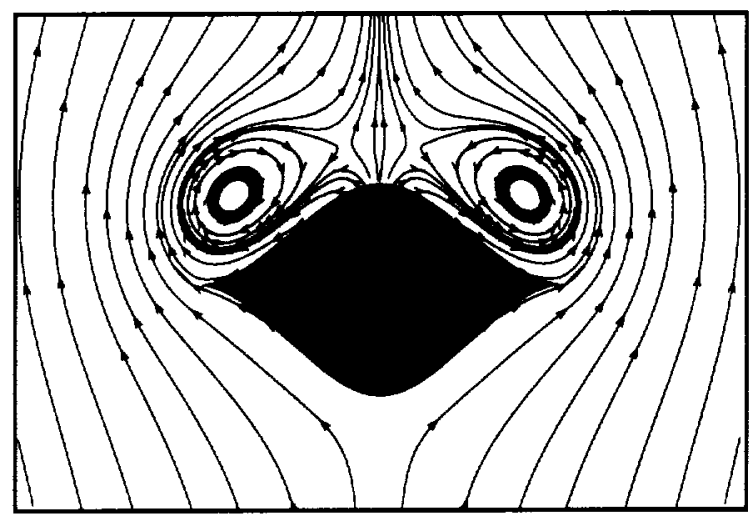

16(a) Baseline Configuration

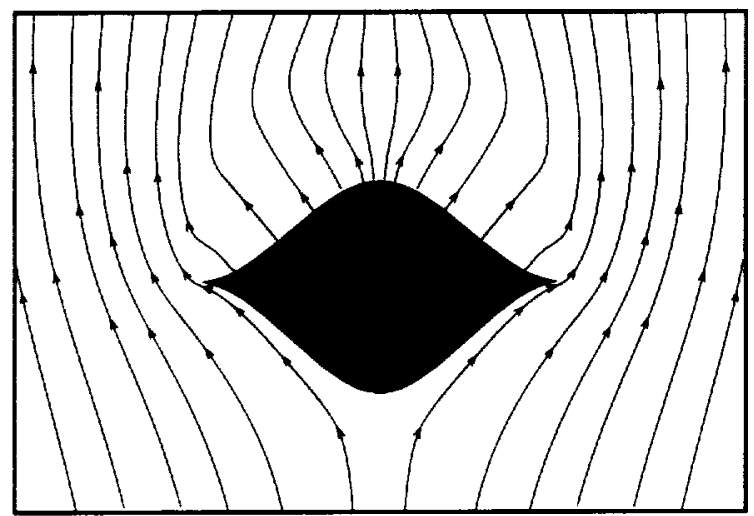

16(b) PI Pitch Control Configuration

Figure 16: Crossflow Streamlines at $F S=100$ 


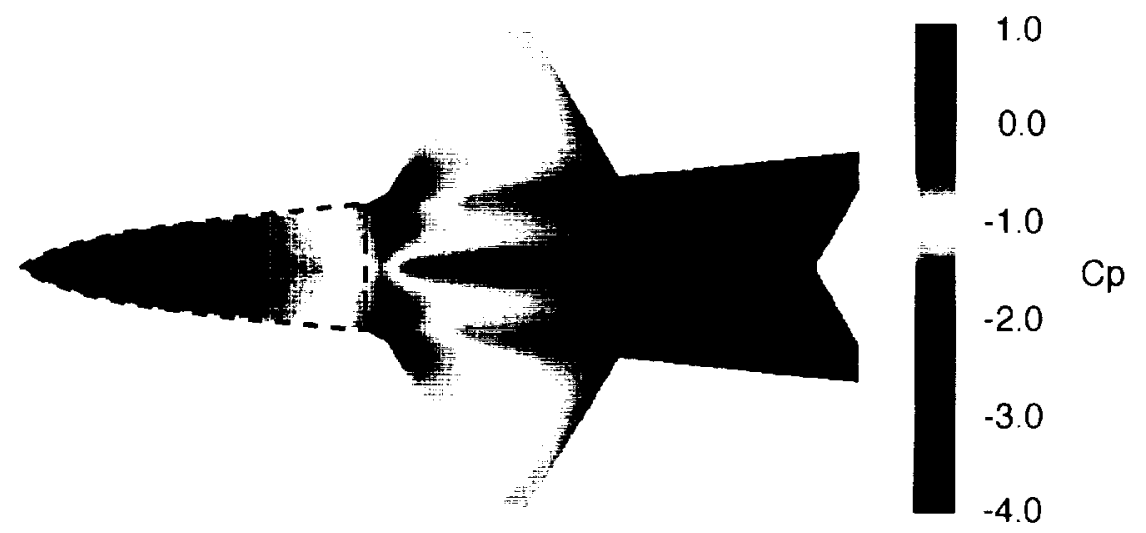

Figure 17: Upper Surface Pressure Coefficient - P2 Pitch Control Configuration

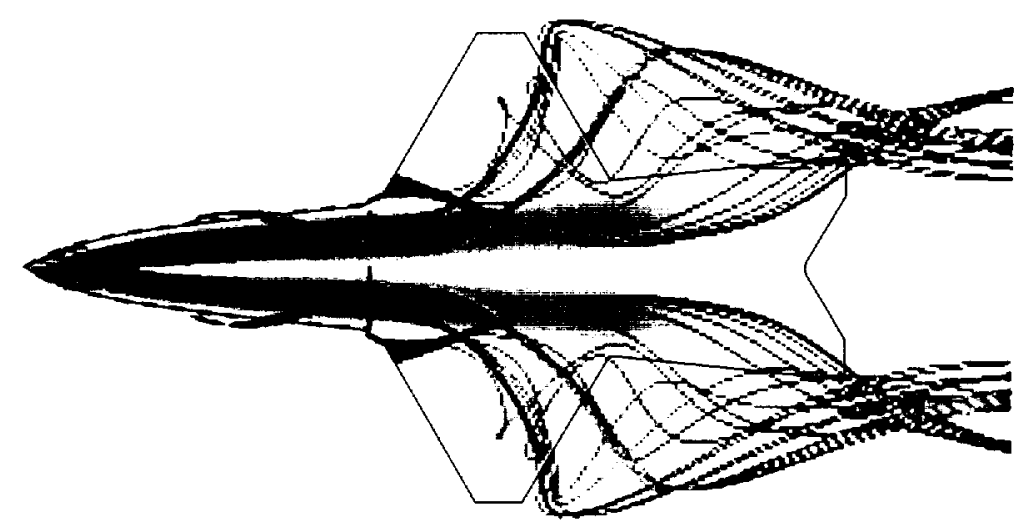

Figure 18: Upper Surface Vortex Flow - P2 Pitch Control Configuration 

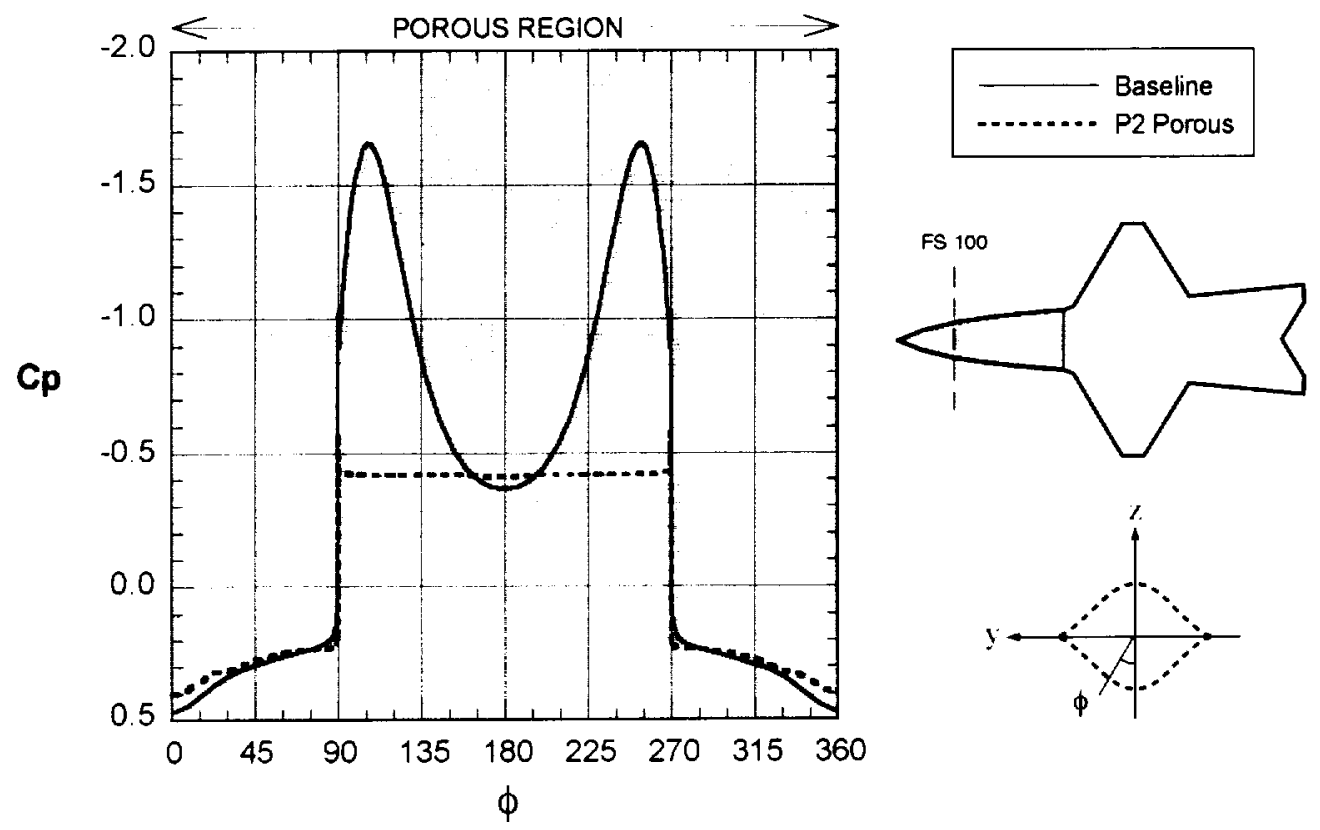

Figure 19: Comparison of Forebody Surface Pressure Coefficient at FS $=100$ Baseline and P2 Pitch Control Configuration

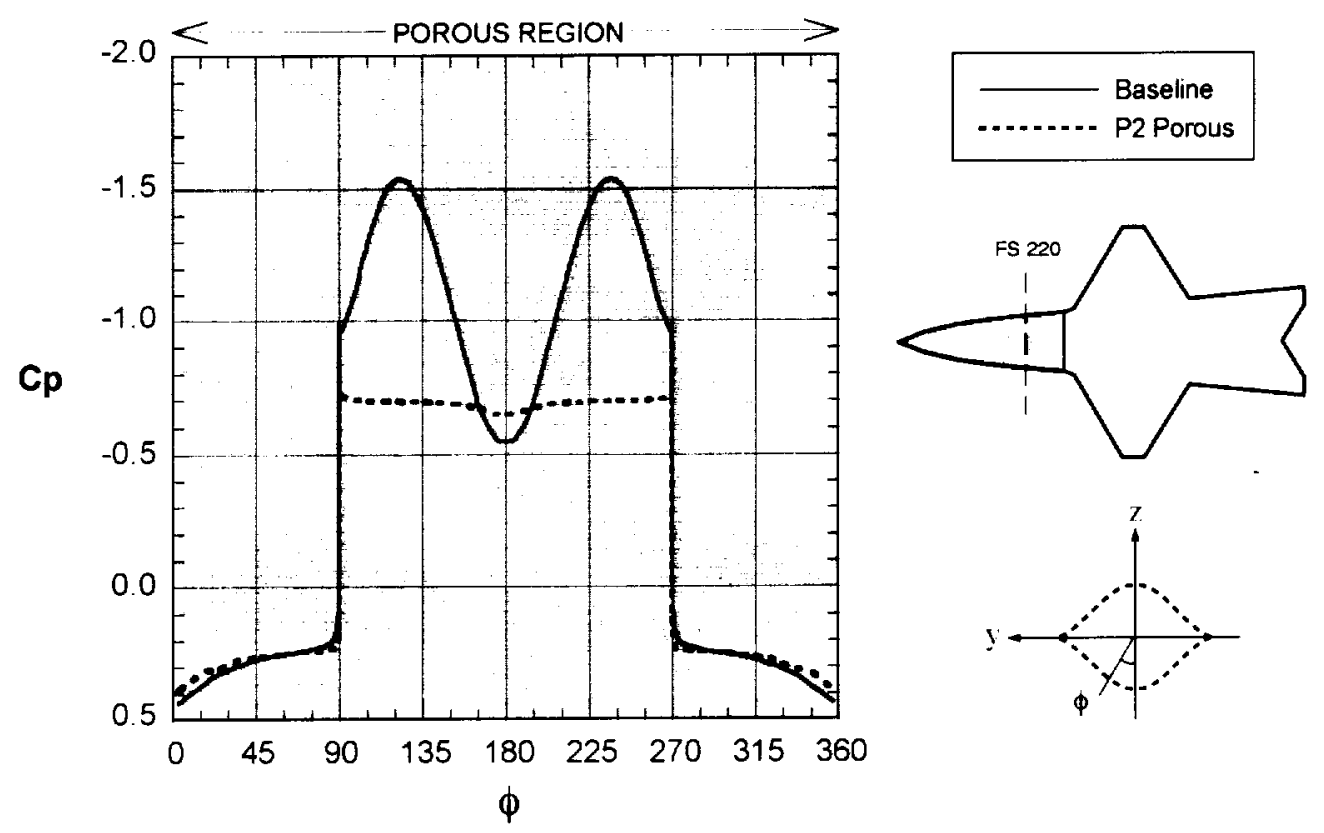

Figure 20: Comparison of Forebody Surface Pressure Coefficient at FS $=220$ Baseline and $P 2$ Pitch Control Configuration 


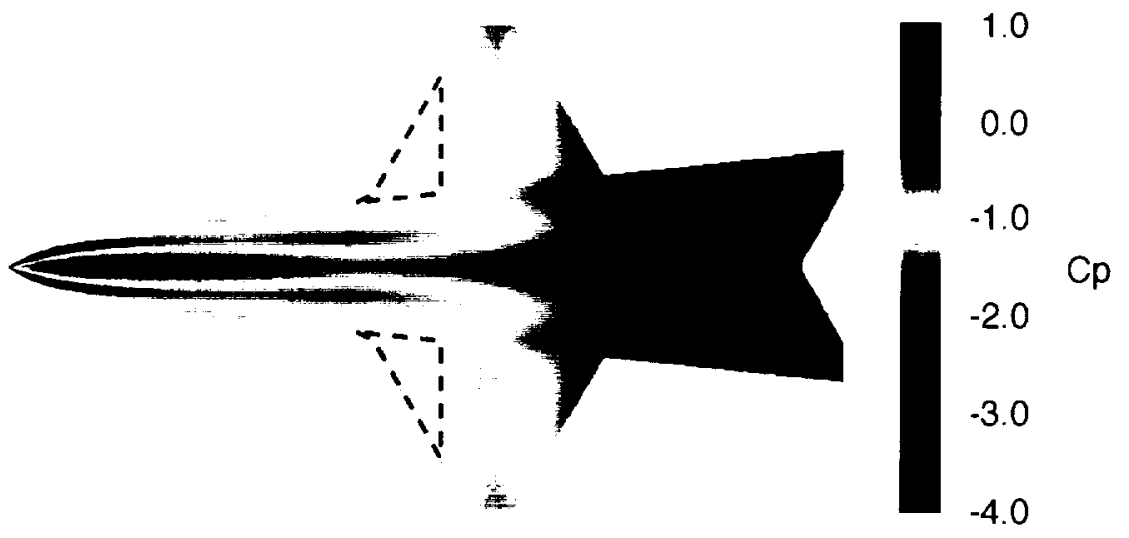

Figure 21: Upper Surface Pressure Coefficient-P3 Pitch Control Configuration

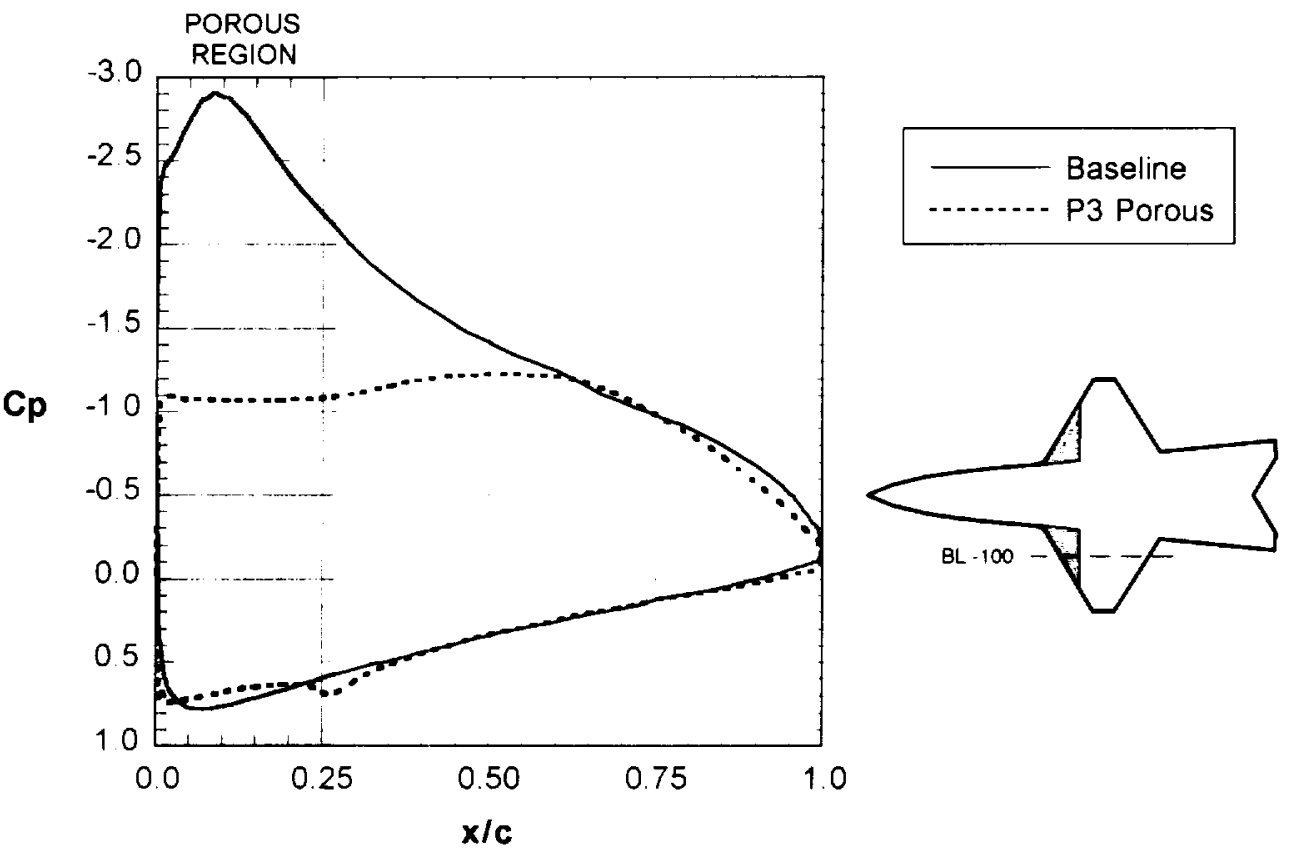

Figure 22: Comparison of Wing Surface Pressure Coefficient at $B L=-100$ Baseline and P3 Pitch Control Configuration

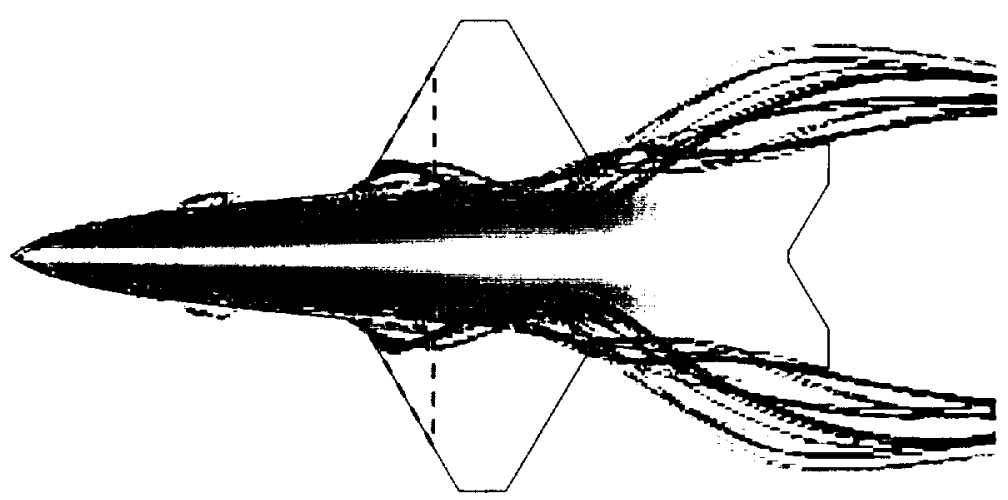

Figure 23: Upper Surface Vortex Flow - P3 Pitch Control Configuration 


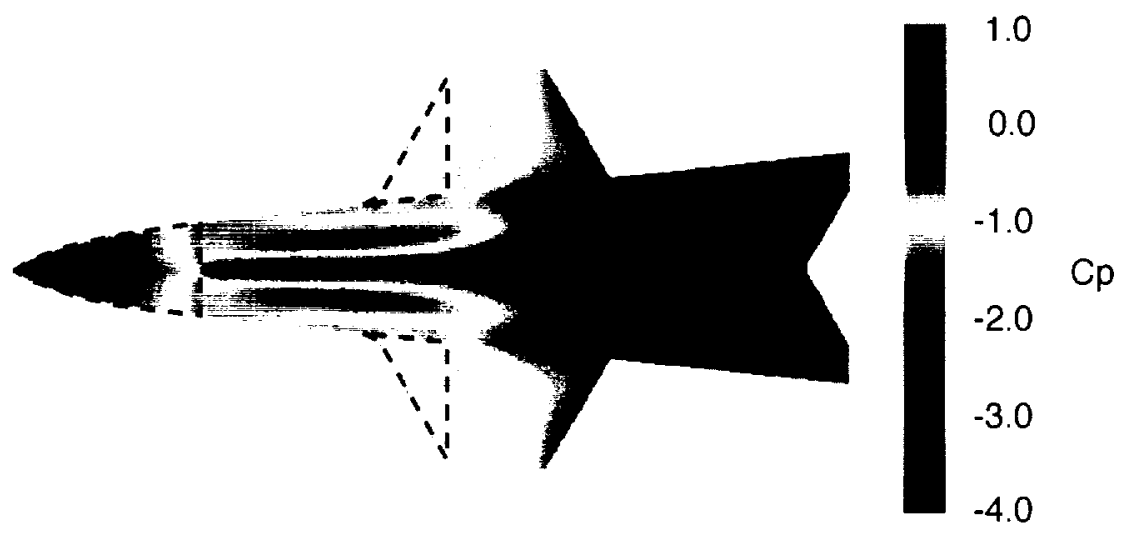

Figure 24: Upper Surface Pressure Coefficient - P4 Pitch Control Configuration

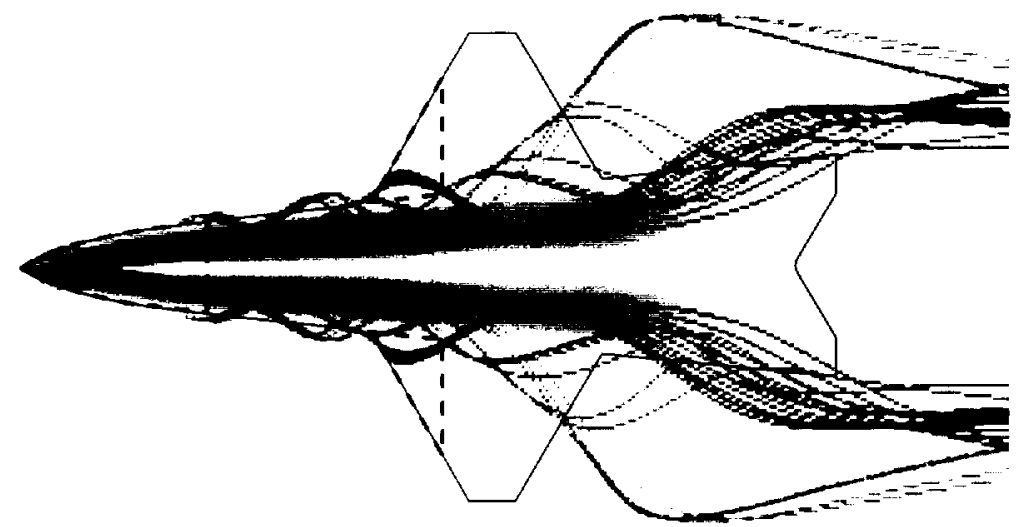

Figure 25: Upper Surface Vortex Flow - P4 Pitch Control Configuration

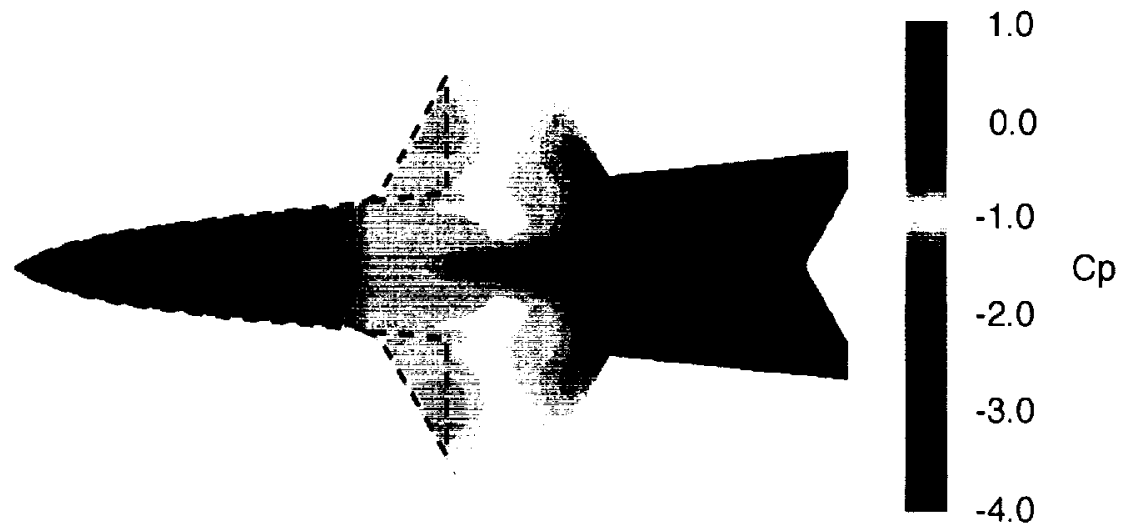

Figure 26: Upper Surface Pressure Coefficient - P5 Pitch Control Configuration 


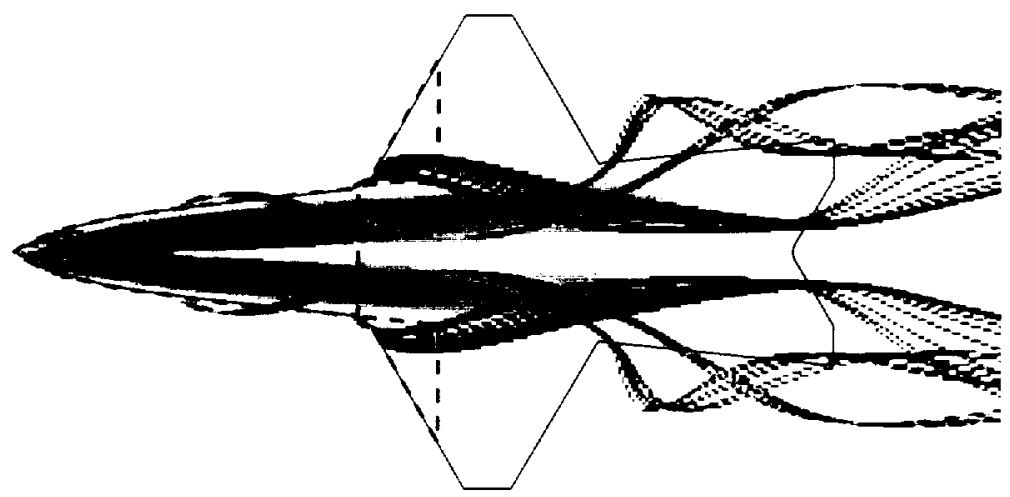

Figure 27: Upper Surface Vortex Flow - P5 Pitch Control Configuration

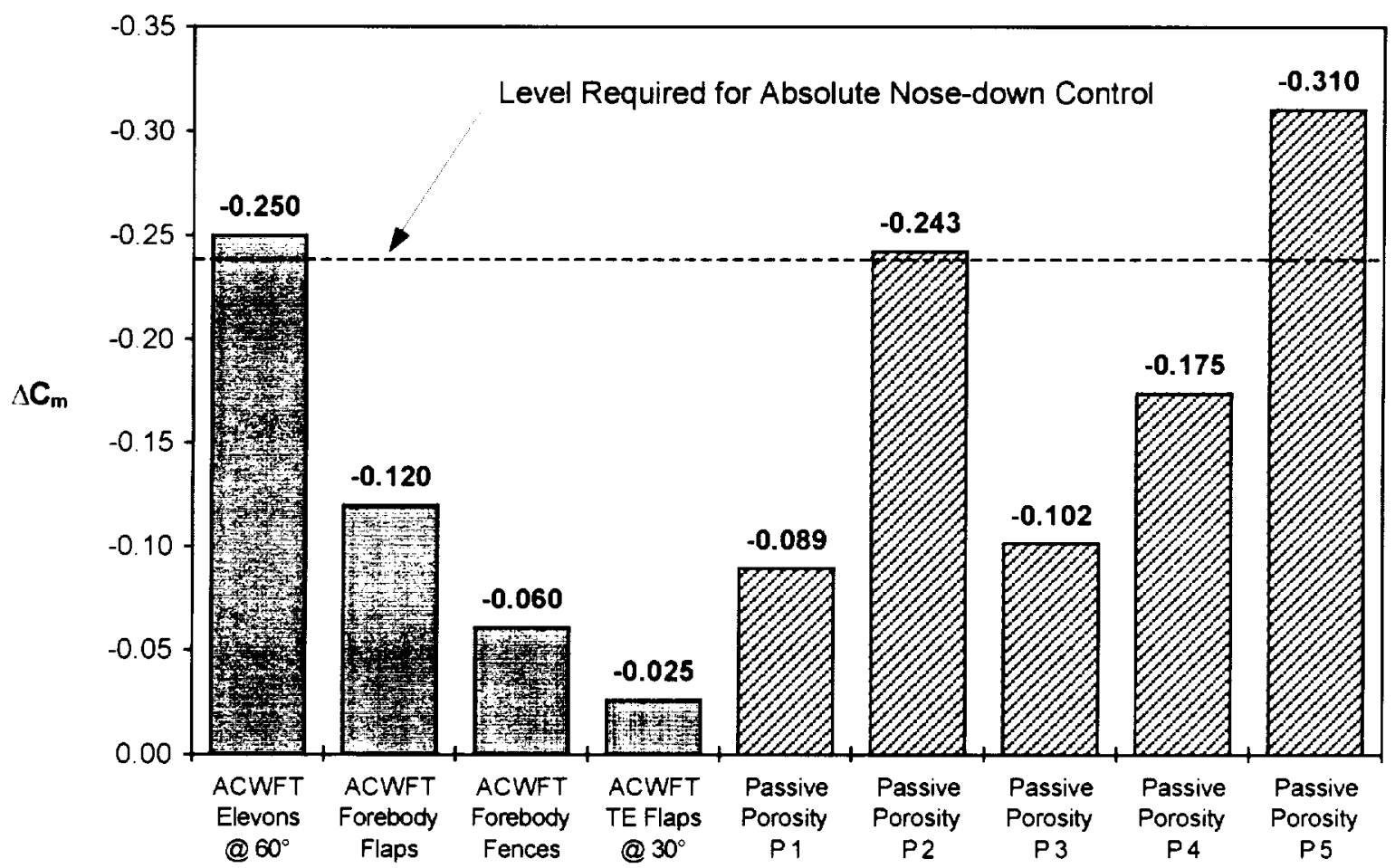

Figure 28: Nose-down Pitch Control Summary 


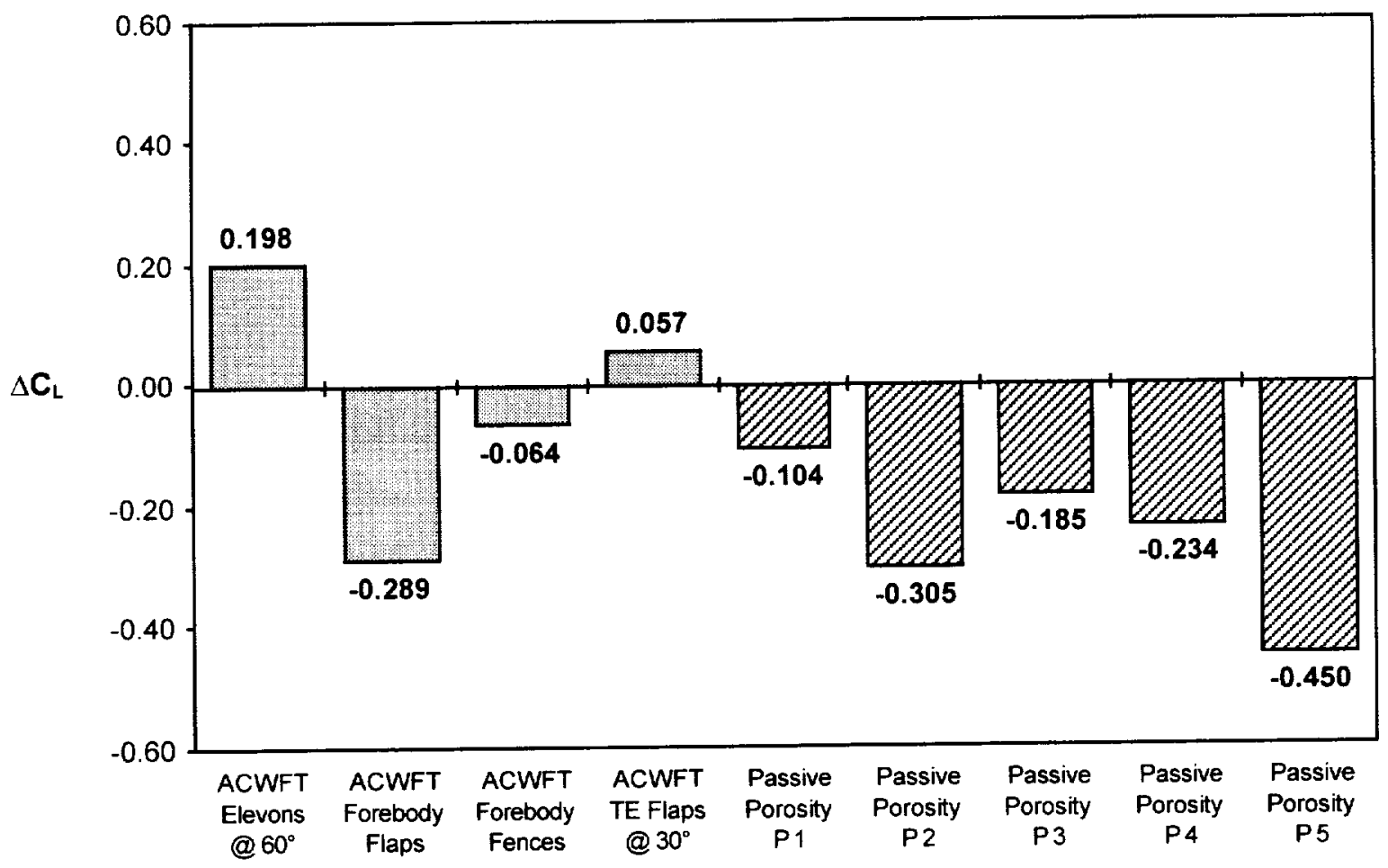

Figure 29: Associated Lift Increments

$-0.4$

$-0.3$

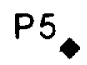

$\mathrm{P} 2$

$\Delta \mathrm{Cm} \quad-0.2$

P4.

$-0.1 * P 3$

P1

0.00

$40 \quad 60 \quad 80 \quad 100$

Percent Porous Region of Forebody

Figure 30: Nose-down Pitch Control Trend

Note: Porous region starts from nose. The baseline and $\mathrm{P} 3$ configurations have $0 \%$ porous forebody area, the $\mathrm{P} 1$ and $\mathrm{P} 4$ configurations have $33 \%$ porous forebody area, and the $\mathrm{P} 2$ and $\mathrm{P} 5$ configurations have $100 \%$ porous forebody area. 

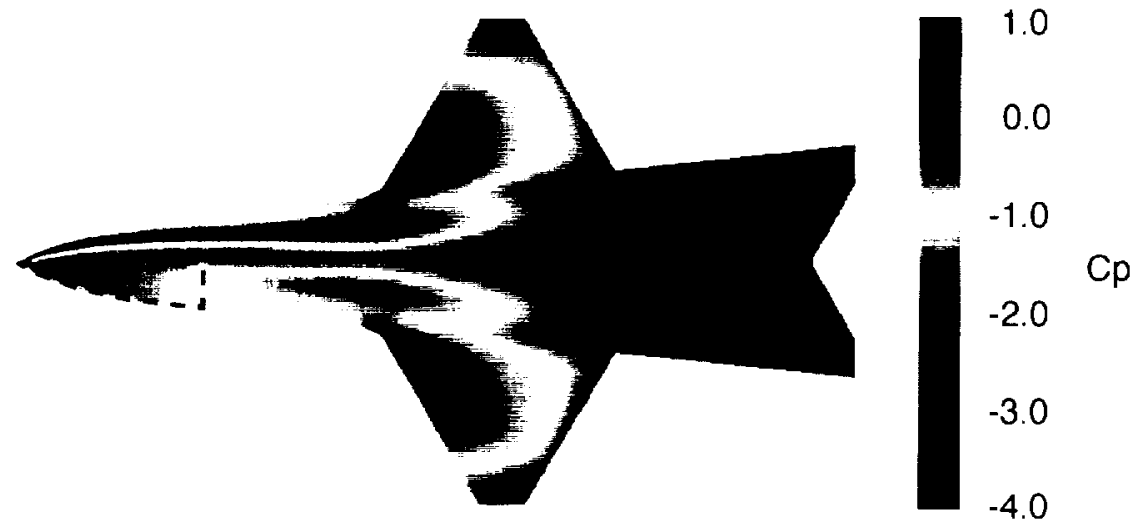

Figure 31: Upper Surface Pressure Coefficient-YI Yaw Control Configuration

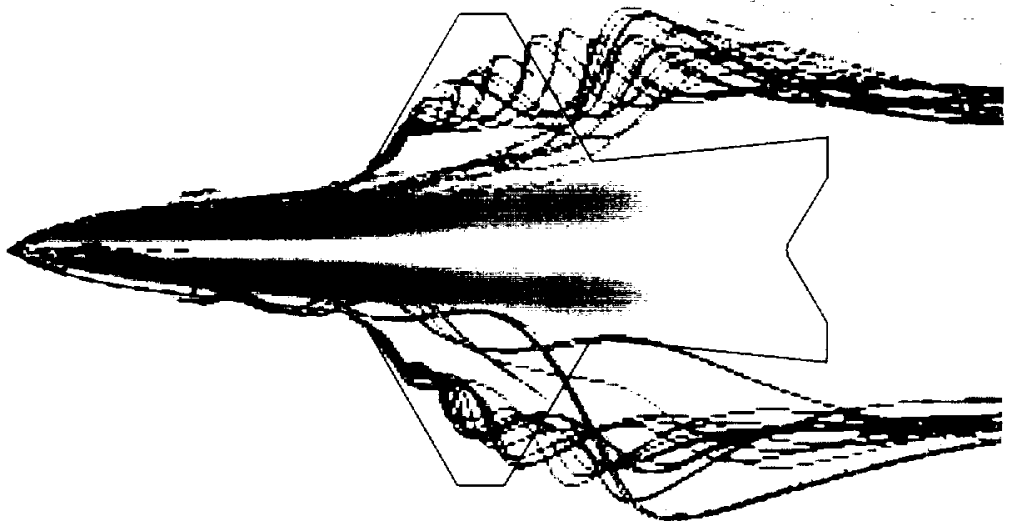

Figure 32: Upper Surface Vortex Flow - Yl Yaw Control Configuration
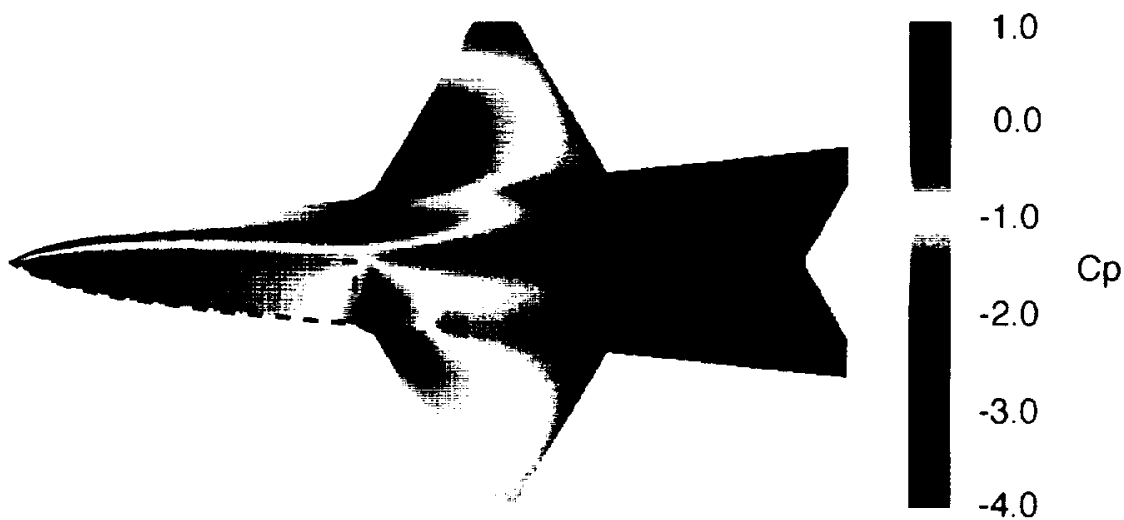

Figure 33: Upper Surface Pressure Coefficient - Y2 Yaw Control Configuration 


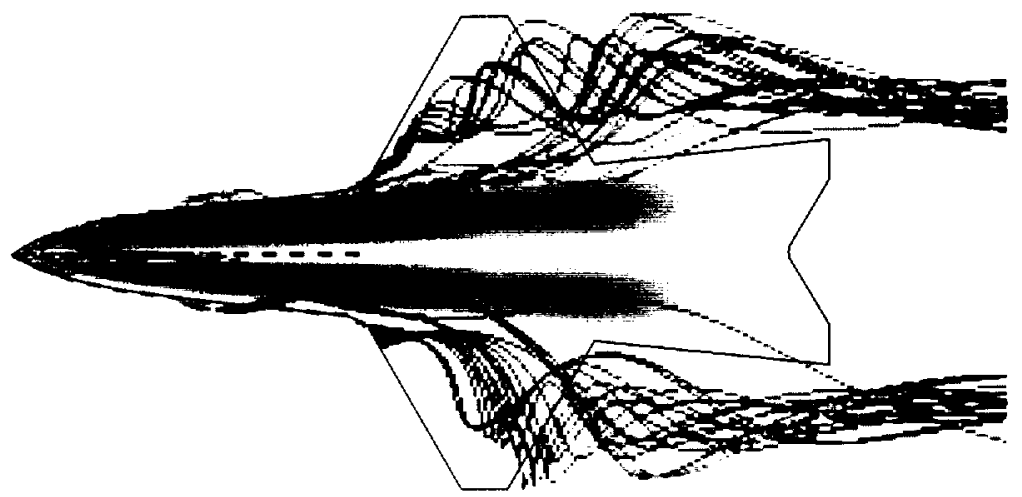

Figure 34: Upper Surface Vortex Flow - Y2 Yaw Control Configuration

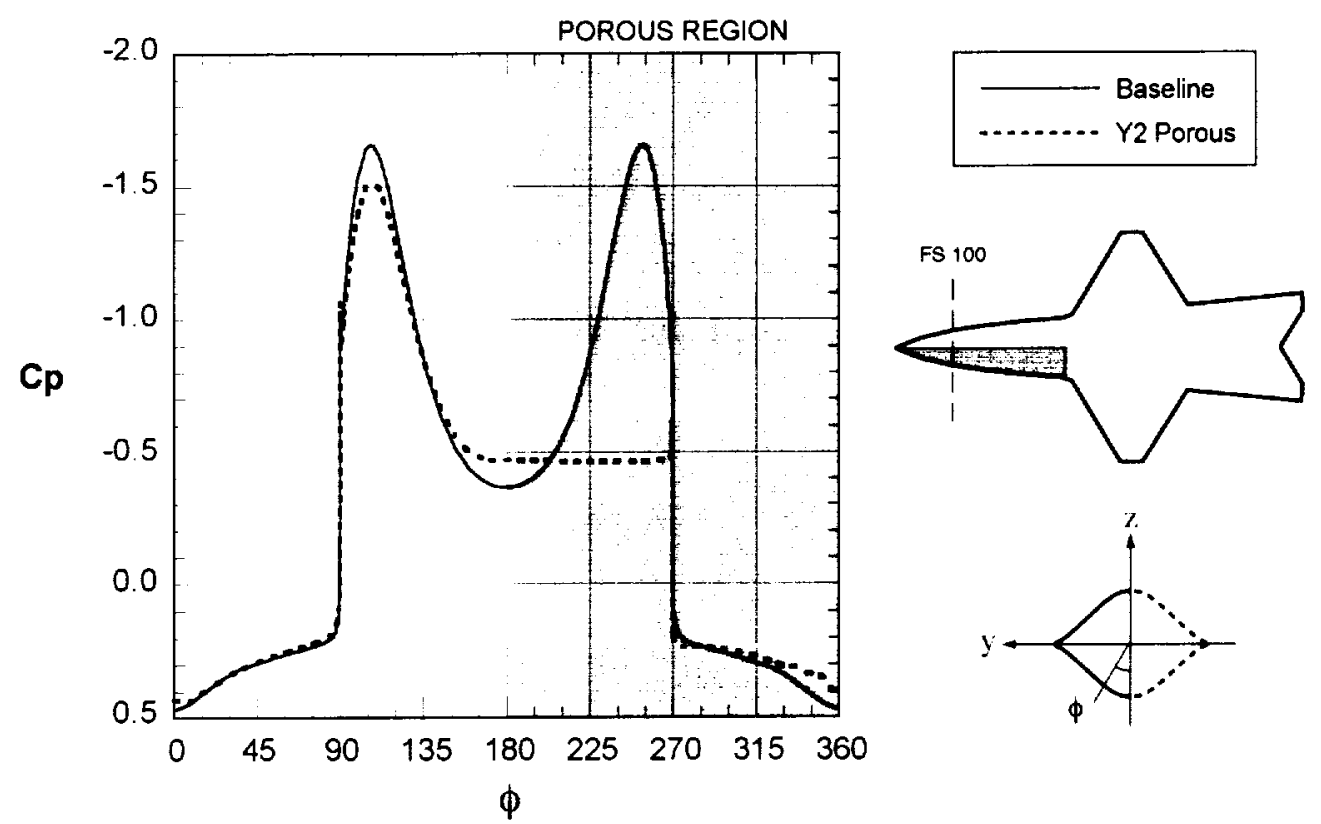

Figure 35: Comparison of Forebody Surface Pressure Coefficient at FS $=100$ Baseline and Y2 Yaw Control Configuration 


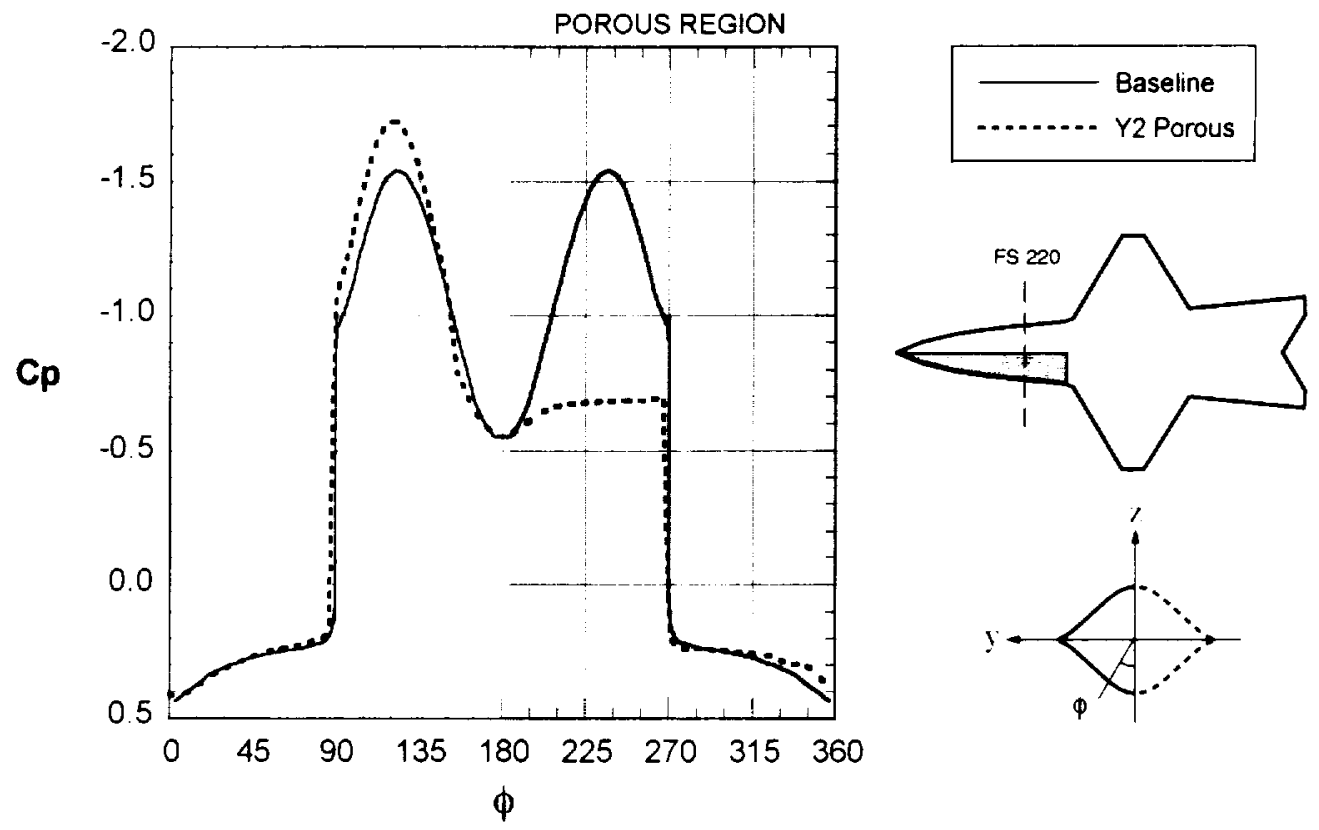

Figure 36: Comparison of Forebody Surface Pressure Coefficient at FS $=220$ Baseline and Y2 Yaw Control Configuration

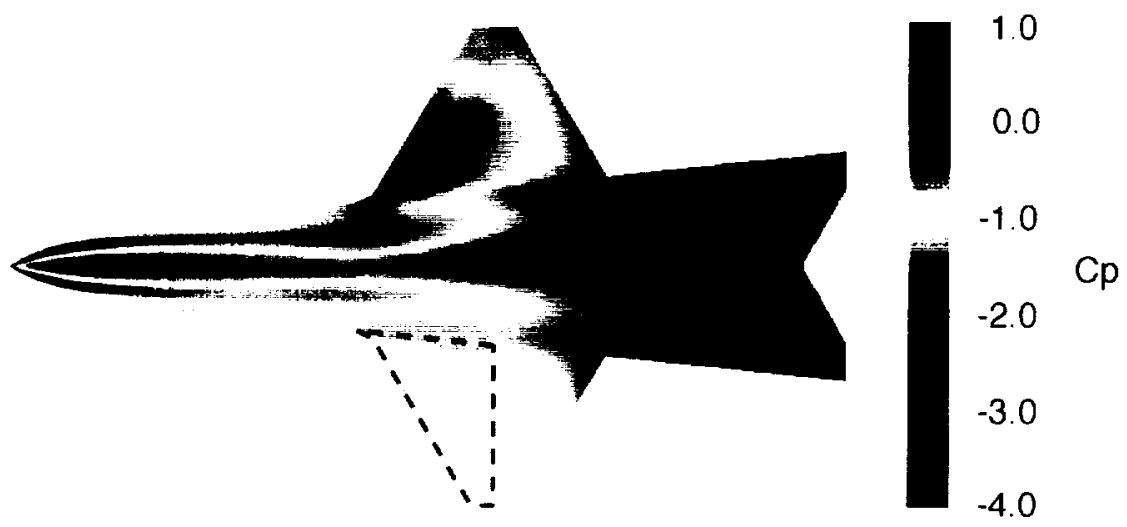

Figure 37: Upper Surface Pressure Coefficient - Y3 Yaw Control Configuration

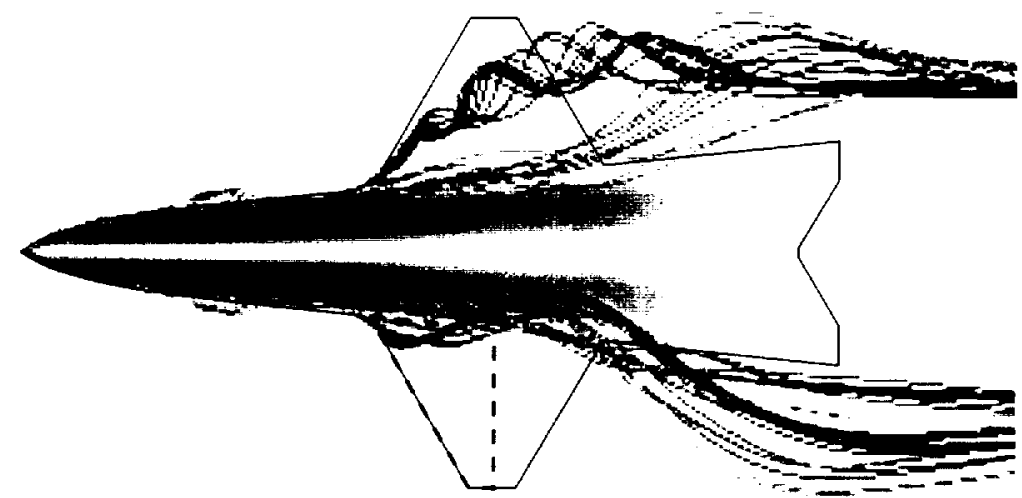

Figure 38: Upper Surface Vortex Flow - Y3 Yaw Control Configuration 


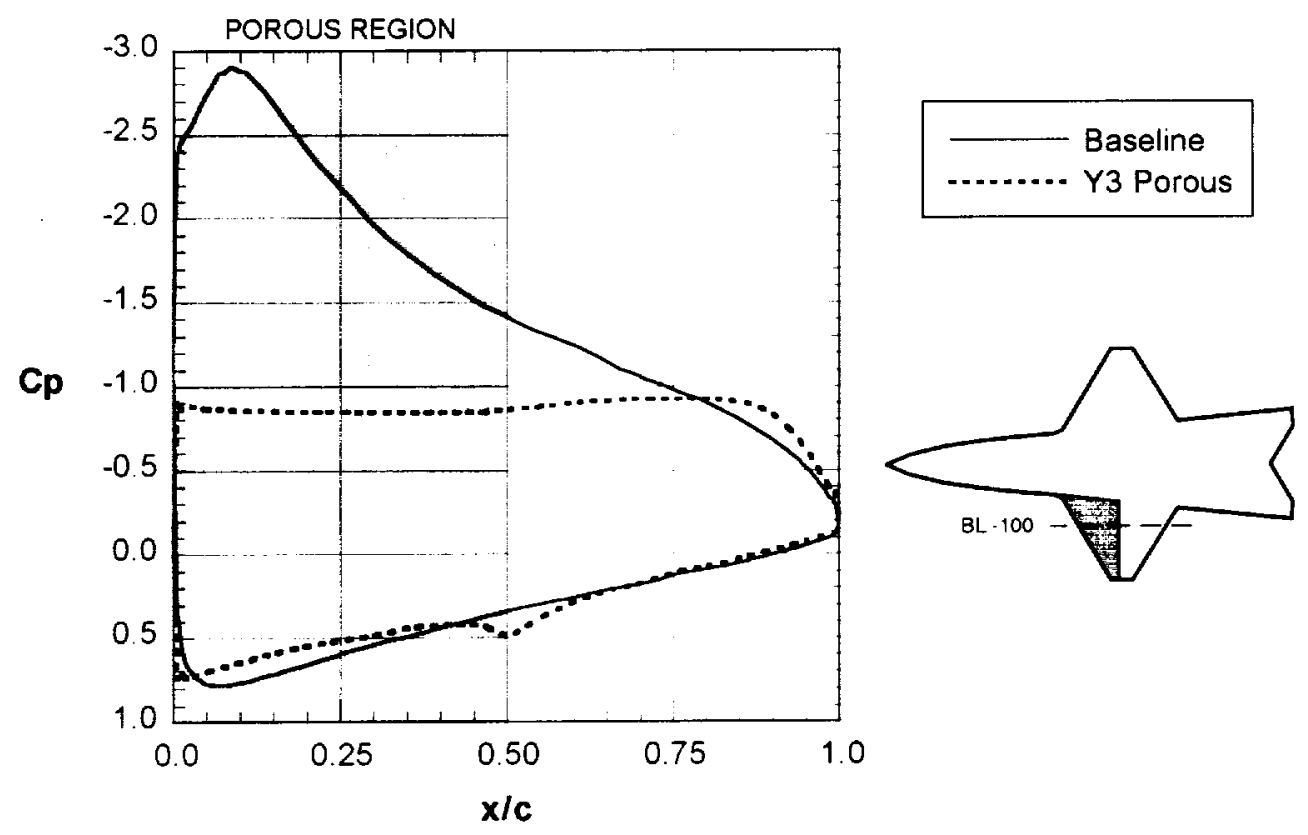

Figure 39: Comparison of Wing Surface Pressure Coefficient at $B L=-100$ Baseline and Y3 Yaw Control Configuration

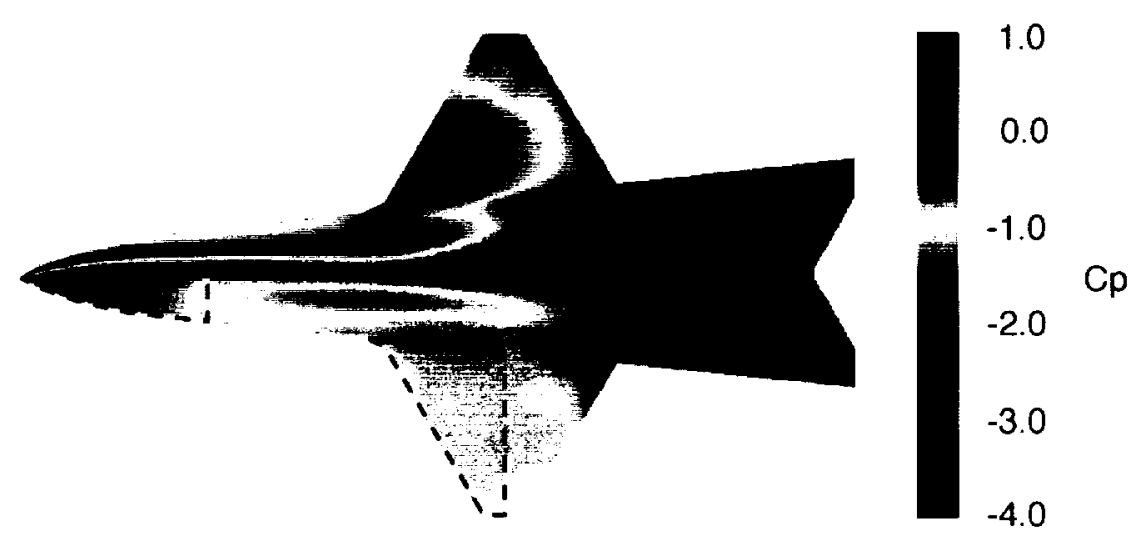

Figure 40: Upper Surface Pressure Coefficient - Y4 Yaw Control Configuration

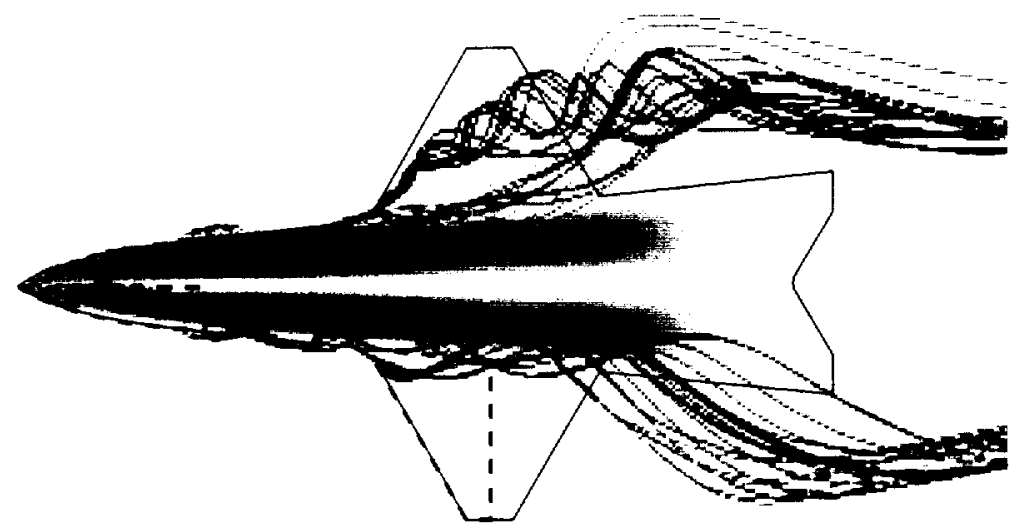

Figure 41: Upper Surface Vortex Flow - Y4 Yaw Control Configuration 


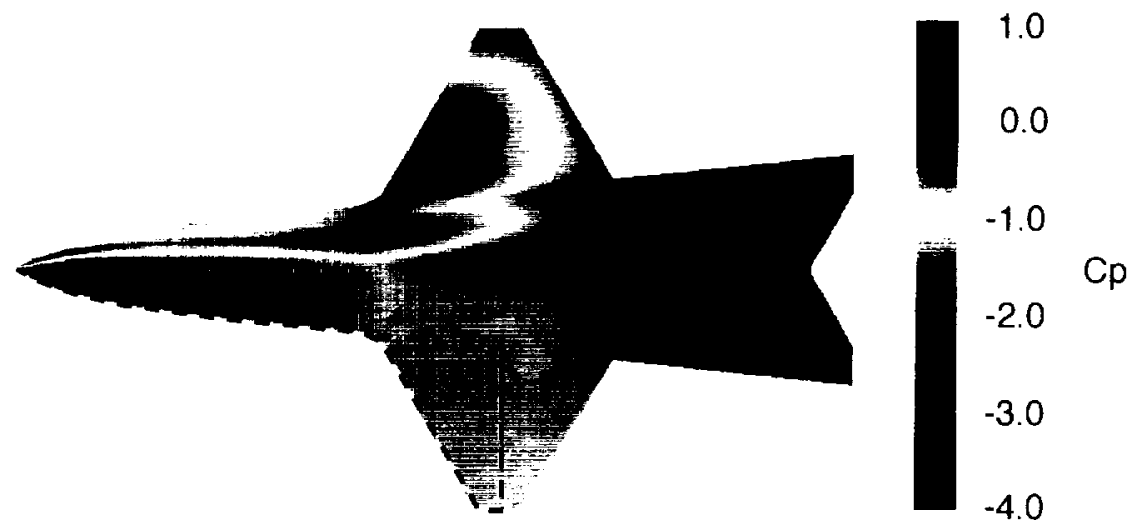

Figure 42: Upper Surface Pressure Coefficient - Y5 Yaw Control Configuration

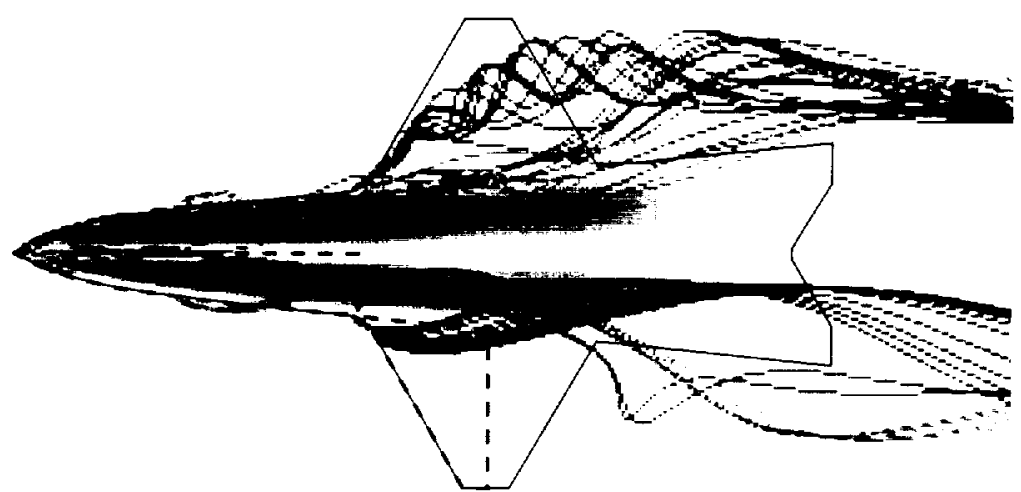

Figure 43: Upper Surface Vortex Flow - Y5 Yaw Control Configuration 


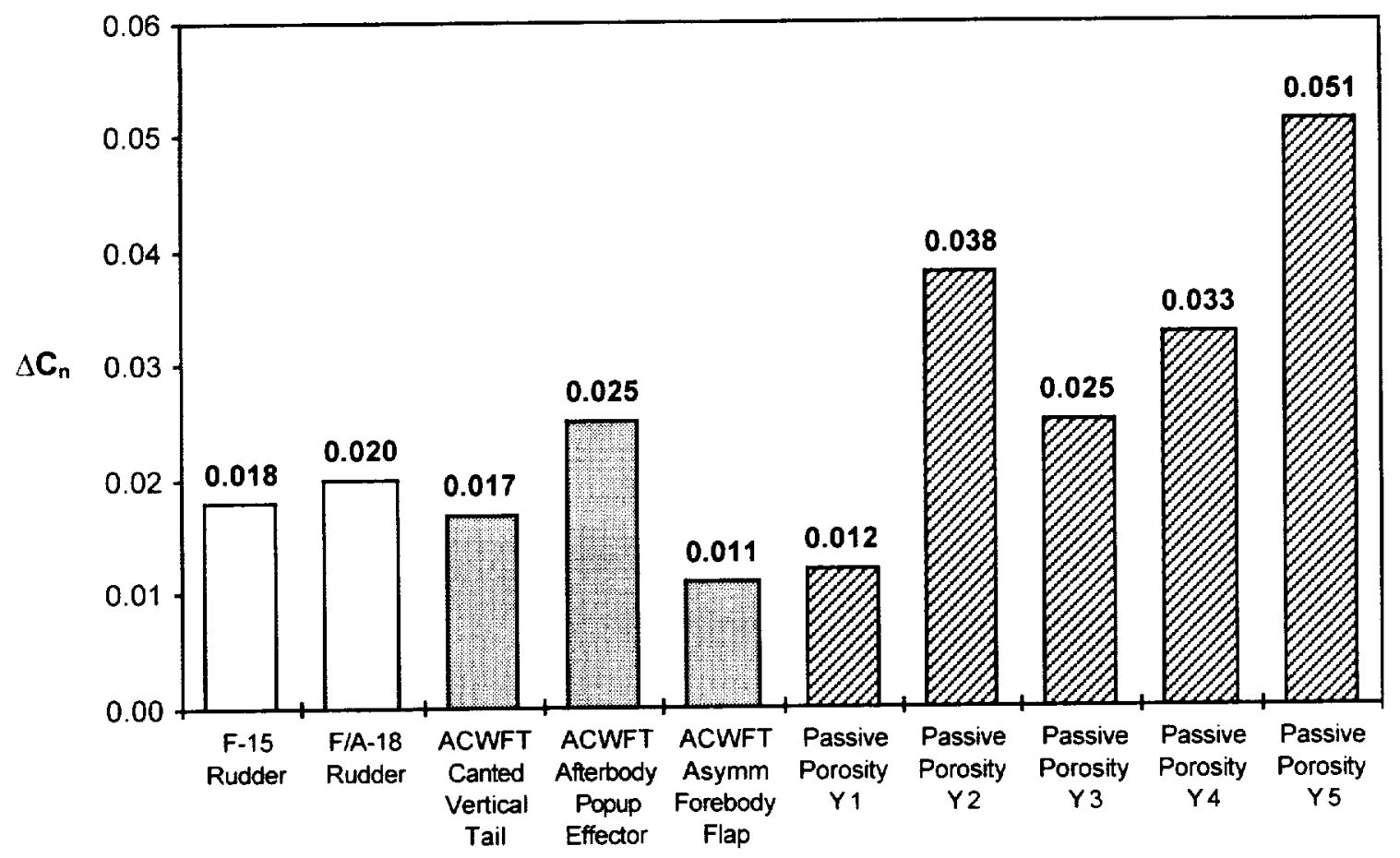

Figure 44: Yaw Control Summary

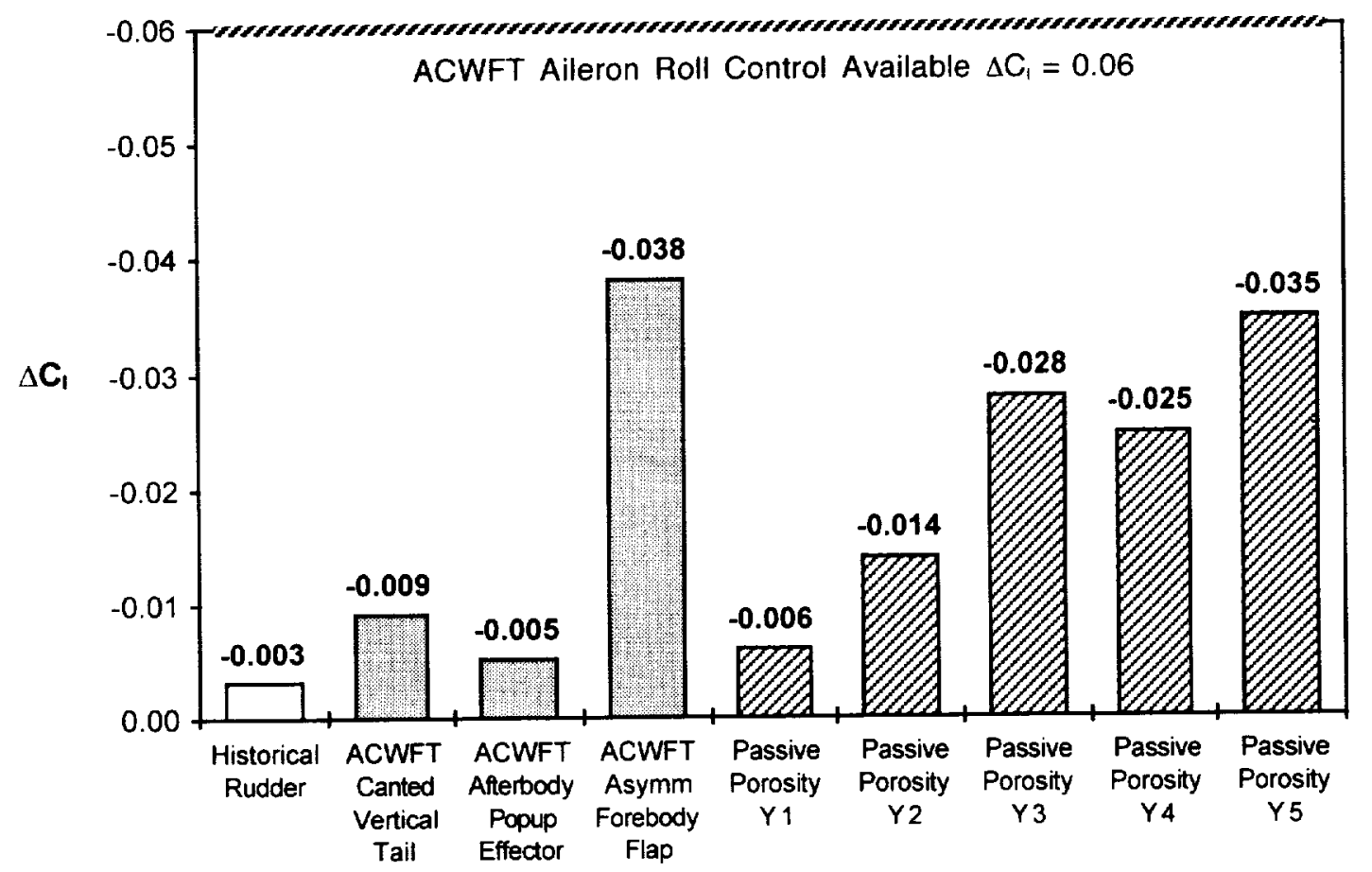

Figure 45: Adverse Roll due to Yaw 


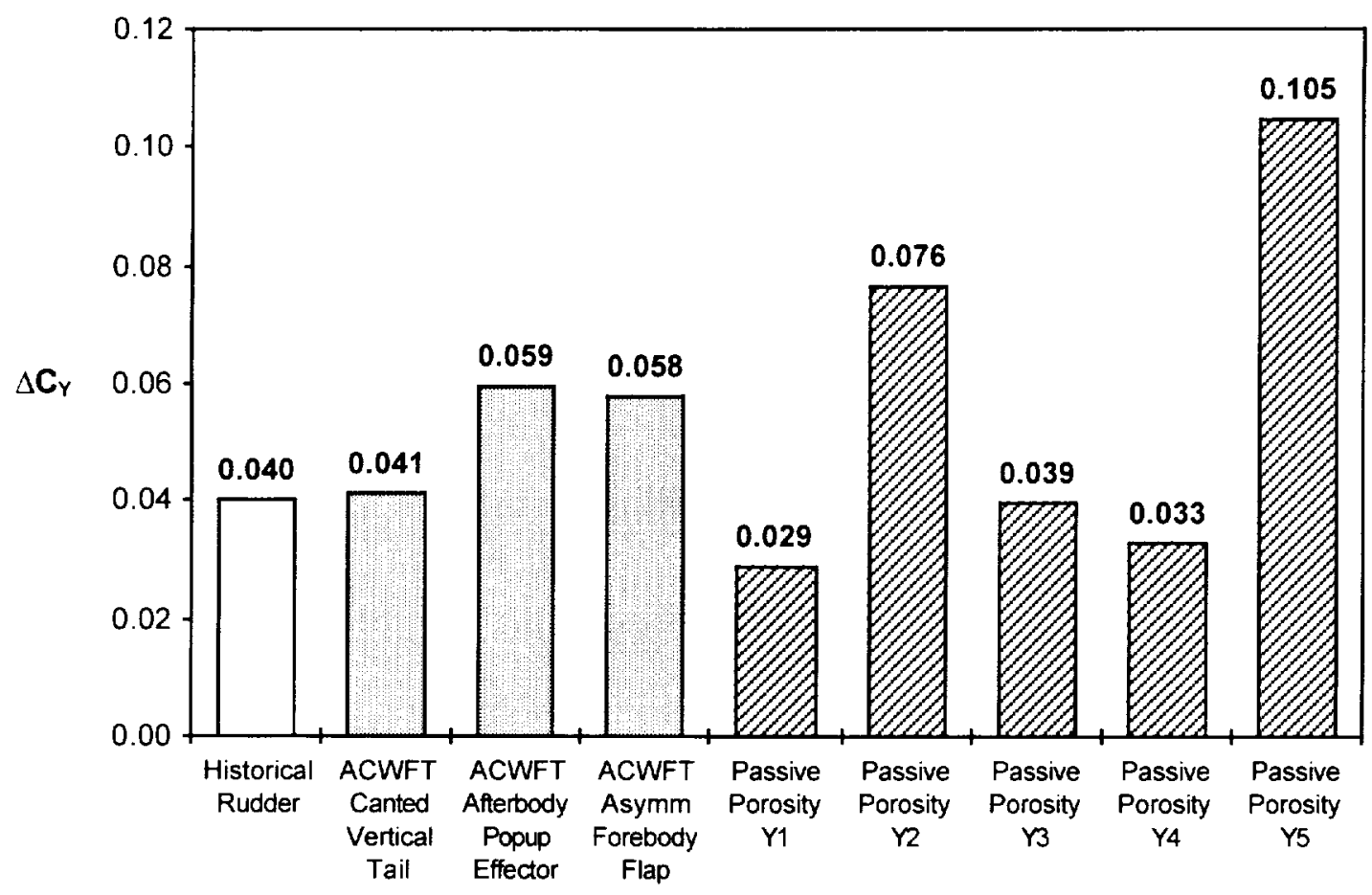

Figure 46: Comparison of Sideforce Increments

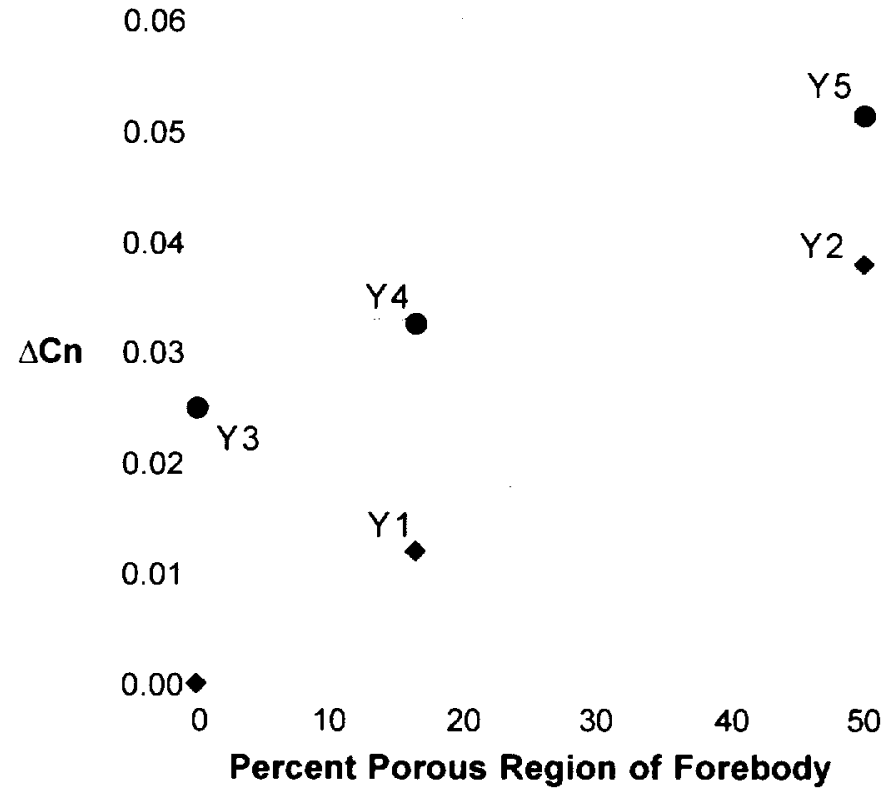

Figure 47: Yaw Control Trend

Note: Porous region starts from nose. The baseline and $\mathrm{Y} 3$ configurations have $0 \%$ porous forebody area, the $\mathrm{Y} 1 / \mathrm{Y} 4$ configurations have $17 \%$ porous forebody area, and the $Y 2 / Y 5$ configurations have $50 \%$ porous forebody area. 

- 